\title{
Nonlinear loop mirror-based all-optical signal processing in fiber-optic communications
}

\author{
Sonia Boscolo, Sergei K. Turitsyn, and Keith J. Blow \\ Photonics Research Group, School of Engineering and Applied Science, Aston \\ University, Birmingham B4 ZET, United Kingdom
}

\begin{abstract}
All-optical data processing is expected to play a major role in future optical communications. The fiber nonlinear optical loop mirror (NOLM) is a valuable tool in optical signal processing applications. This paper presents an overview of our recent advances in developing NOLM-based all-optical processing techniques for application in fiber-optic communications. The use of in-line NOLMs as a general technique for all-optical passive $2 \mathrm{R}$ (reamplification, reshaping) regeneration of return-to-zero (RZ) on-off keyed signals in both high-speed, ultralong-distance transmission systems and terrestrial photonic networks is reviewed. In this context, a theoretical model enabling the description of the stable propagation of carrier pulses with periodic all-optical self-regeneration in fiber systems with in-line deployment of nonlinear optical devices is presented. A novel, simple pulse processing scheme using nonlinear broadening in normal dispersion fiber and loop mirror intensity filtering is described, and its employment is demonstrated as an optical decision element at a RZ receiver as well as an in-line device to realize a transmission technique of periodic all-optical RZ-non-return-to-zero-like format conversion. The important issue of phase-preserving regeneration of phase-encoded signals is also addressed by presenting a new design of NOLM based on distributed Raman amplification in the loop fiber.
\end{abstract}

Key words:

Nonlinear fiber optics, Optical fiber communications, Optical signal processing, Optical regeneration

PACS: 42.65.Hw, 42.81.Uv, 42.79.Sz, 42.79.Hp

Email address: s.a.boscolo@aston.ac.uk, s.k.turitsyn@aston.ac.uk, k.j.blow@aston.ac.uk (Sonia Boscolo, Sergei K. Turitsyn, and Keith J. Blow). 


\section{Introduction}

The use of photonic technologies for data processing in the all-optical domain is a field of growing importance, with a strong potential for a variety of interesting applications in such diverse areas as ultrahigh-speed optical telecommunications, ultrafast metrology, optical sensing, microwave engineering, image processing and optical computing. Advantages of processing the information in the all-optical domain include the tremendous available bandwidth and the parallelism intrinsic to the optical approach, which translate into ultrahigh processing speeds that are not possible otherwise. In the present optical communcation systems, signal processing functions such as switching, routing, frequency conversion, and regeneration are typically performed electrically, which requires converting the optical signal to electrical and back to optical again for retransmission. While this traditional approach has the advantage of relying on mature technology, there is significant interest in bypassing the optoelectronic conversion and undertaking processing tasks entirely in the optical domain, since the all-optical approach provides a route to optical networks of increased capacity, scalability, and flexibility, as well as reduced network management complexity and costs. Among the all-optical signal processing technologies, all-optical regeneration is considered a key-enabling function for the deployment of future all-optical communication systems as a means of restoring the quality of the signals, which is deteriorated by impairments such as noise accumulation from optical amplifiers, fiber chromatic dispersion, different types of crosstalk, jitter and nonlinearities, and other imperfections in transmission and cross-connects [1,2]. Although ultimately reamplification, reshaping and retiming (3R regeneration) of the signals is required, for many applications simple and cost-efficient $2 \mathrm{R}$ regenerators (devices with reamplification and reshaping characteristics only) can be an attractive practical solution. The central component of most all-optical regenerators is a nonlinear optical gate, which perform the decision in the optical domain. This gate is most commonly realized through nonlinear phenomena in semiconductors and optical fibers. The semiconductor-based devices exploiting cross-gain/absorption/phase-modulation in a semiconductor optical amplifier (SOA), a saturable absorber, and an electroabsorption modulator [3-13] are compact in size and probably most suitable for mass production. The operating speed of such devices, however, is often dominated by the carrier life time. Slow carrier recovery causes significant pattern effect, which can be a serious problem in regeneration applications since it rather degrades the quality of the recovered signal. On the other hand, for the fiber-based devices incuding those utilizing four-wave mixing (FWM) [14-18], self-phase and cross-phase modulation [19-29], and nonlinear interferometry within an optical loop mirror [30-47], the operating speed is determined by ultrafast fiber nonlinearity, which provides response times in the femtosecond range. But the fiber-based devices are typically larger in size compared with the SOA-based ones. This, 
however, is being improved with the recent developments of highly nonlinear fibers (HNLFs) [48], which enable the implementation of short devices operated at reduced powers. The intrinsic simplicity and large speed potential of the fiber-based regenerators appear fascinating for the realization of all-optical regeneration in future photonic networks.

Owing to its self-switching ability and flexibility, the fiber Sagnac interferometer or nonlinear optical loop mirror (NOLM), originally proposed in [49], has been recognized as a valuable tool in a large field of optical signal processing applications. These include optical switching [50-55], phase conjugation [56], wavelength demultiplexing [57-59], mode-locking [60-64], pulse compression and pedestal suppression [65-69]. Earlier studies [70-74] have addressed the use of NOLMs to reshape and stabilize soliton pulses. More recent studies [30$47,75,76]$ have established the NOLM as an effective $2 \mathrm{R}$ regenerative device in telecommunication systems. The NOLM, made of a coupler whose output ports are connected through a span of fiber, offers a versatile way of obtaining a nonlinear transmission (or switching) characteristic through the optical Kerr effect, if a nonlinear phase shift difference appears between the two interfering beams. A variation of the differential phase by $\pi$ will cause a change of the NOLM from high transmission to a high reflection. Most NOLM designs rely on self-phase modulation (SPM), which allows a nonlinear phase shift to accumulate if a power imbalance exists between the counterpropagating beams in the loop. Breaking of the loop power symmetry can be done with an asymmetric coupler [49], or alternaltively with a gain or loss element spliced asymmetrically within the loop [77], or alternatively by making the dispersion of the fiber asymmetric [66]. A variation of the NOLM design uses polarization asymmetry between the counterpropagating beams to achieve switching through nonlinear polarization rotation [54]. The NOLM typically exhibits a sinusoidal nonlinear transmission characteristic. Low transmission at low power enables suppression of noise and radiative backgruond, whereas stable fixed points of the input-output power mapping can be used to reduce the amplitude jitter of the carrier pulses.

In this paper, we report on our recent results on the development of alloptical signal processing techniques utilizing the NOLM for application in fiber-optic communications. In Section 2, we describe the main results of the application of in-line NOLMs as a general technique for all-optical passive $2 \mathrm{R}$ regeneration of return-to-zero (RZ) on-off keyed (OOK) signals in both highspeed, ultralong-distance transmission systems [30-36] and terrestrial photonic networks $[37,38]$. A theoretical model enabling the description of the stable propagation of ultrashort optical pulses in fiber transmission systems in the quasi-linear regime, with lumped nonlinear optical devices (NODs) placed periodically along the line $[78,79]$ is presented in Section 3, and applied to a system with in-line NOLMs. In Section 4 we describe a novel, simple pulse processing scheme for use at a RZ optical receiver, which is based on nonlinear 
temporal pulse broadening in a normal dispersion fiber (NDF) and loop mirror intensity filtering [80-82]. A novel transmission technique based on periodic inline all-optical format conversion between RZ and non-return-to-zero (NRZ)like is also demonstrated by use of such NDF-enhanced NOLMs [83]. In Section 5 , we present a concept for phase-preserving all-optical $2 \mathrm{R}$ regeneration of signals using phase-shift keying (PSK) modulation formats, which exploits a new design of NOLM based on distributed Raman amplification in the loop fiber (RA-NOLM) [84]. The regenerative scheme is applied to RZ differential PSK (DPSK) transmission. Section 6 summarizes the results presented in this review.

\section{All-optical $2 \mathrm{R}$ regeneration of OOK signals}

In this section, after reviewing the basic properties of the NOLM, we demonstrate that the in-line deployment of NOLMs is an effective technique of alloptical 2R regeneration of RZ-OOK signals in high-speed, strongly dispersionmanaged (DM) fiber communication systems.

\subsection{Configuration and operation principle of the NOLM}
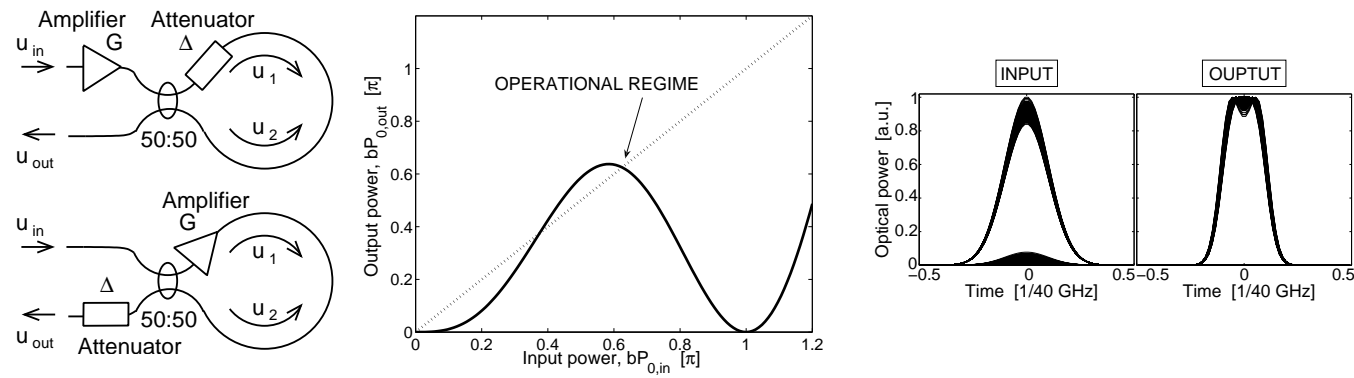

Fig. 1. Left, scheme of the NOLM: loss-imbalanced (top) and gain-imbalanced (bottom) configurations. Center, CW power characteristic. Right, optical signal eye-diagrams at the NOLM input and output.

Here and hereafter, we use a loss or gain-imbalanced NOLM as depicted in Fig. 1 (left). A preamplifier or postattenuator is incorporated in the NOLM setup. Note that in the practical implementation of a regenerated transmission system, pulse preamplification can be achieved by addition of an extra gain to the line amplifier immediately preceding the NOLM [36-38]. An incoming signal $u_{\text {in }}$ is splitted by a 50:50 coupler into two partial signals $u_{1}$ and $u_{2}$, which counterpropagate in the fiber loop. As a result of the discrimination provided by the loop attenuator or amplifier, the two signals gather different power levels and, thus, different phase shifts induced by the fiber Kerr nonlinearity through SPM before they interfere at the coupler. This interference leads to a 
power-dependent amplitude transfer function $f(P) \equiv u_{\text {out }} / u_{\text {in }}$, which can be written in the form

$$
f(P)=a \sin (b P) \exp (i c P) .
$$

An unimportant phase factor is omitted from Eq. (1), and the effect of dispersion in the fiber is also neglected. The parameters $a, b$, and $c$ read: $a=$ $\sqrt{G \Delta} \exp (-\gamma L), b=\sigma G(1-\Delta) L_{\text {eff }} / 4$ or $b=\sigma(G-1) L_{\text {eff }} / 4$, and $c=$ $\sigma G(1+\Delta) L_{\text {eff }} / 4$ or $c=\sigma(G+1) L_{\text {eff }} / 4$. Here, $\sigma$ is the nonlinear coefficient of the loop fiber, $\Delta$ is the loss coefficient of the loop attenuator or postattenuator, $G$ is the gain coefficient of the preamplifier or loop amplifier, $\gamma=0.05 \ln (10) \alpha$ is the fiber loss coefficient with $\alpha$ in $\mathrm{dB} / \mathrm{km}, L$ is the length of the loop fiber, and $L_{\text {eff }}=(1-\exp (-2 \gamma L)) /(2 \gamma)$. The (normalized) continuous-wave (CW) power mapping curve according to (1) is plotted in Fig. 1 (center). We operate the system such that the peak power $P_{0 \text {,in }}$ of the input pulses is slightly past the first peak of the CW characteristic curve [31-36]. A CW analysis shows that this region permits operational points that are stable against small fluctuations in the input power $[65,79]$. We also require equalization of the pulse peak power at the input and the output of the system. We then obtain $2 b P_{0, \text { in }}=(1+\epsilon) \pi, 0<\epsilon<1$, and $a \cos (\epsilon \pi / 2)=1$ from Eq. (1). The solution of these equations for given $P_{0 \text {,in }}$ and $\epsilon$, and fixed fiber parameters, provides two of the parameters $\Delta, G$ and $L$ in terms of the third one, thus enabling device scalability. It is useful to note here that short fiber lengths are desirable for improving the stability and polarization insensitivity of the loop mirror. Indeed, to implement practical NOLM-based regeneration, vibration-insensitive and polarization-independent operation is indispensable. The necessary fiber length to switch the loop mirror into transmittance can be made sufficiently short by use of a HNLF [43-46]. In our approach, however, the loop length may be changed at will since changing $L$ is just a matter of rescaling $\Delta$ and $G$. We also mention that a simple method to overcome the polarization sensitivity of the NOLM is twisting the loop fiber to generate circular birifringence [58], and stable operation against external vibration can be realized by the use of a polarization-rotating mirror [85].

The principle of the regeneration function of the NOLM is as follows: lowintensity noise and dispersive waves are filtered out from the higher-power pulses through an effective saturable absorption action [65]. This allows for restoration of the pulse amplitude and shape [30-36]. Also, operation of the NOLM just after the peak of its switching curve enables stabilization of the pulse amplitude against fluctuations in the input power [31-36]. Note that the loss or gain-imbalanced NOLM provides higher discrimination against lowpower radiation than a coupler-imbalanced NOLM [71] owing to its approximate amplitude cubing effect at low powers (see (1)). On the contrary, the transfer function of a coupler-imbalanced NOLM [65] rises linearly at low powers. But since the loss or gain-imbalanced NOLM has sharper swtiching peaks, the portion of the switching curve on which operation is stable is smaller. However, this limitation can be overcome by placing the operation point only 
slightly past the switching peak. A strongly coupler-imbalanced NOLM has been investigated in $[40,41]$. This device suppresses rather large pulse amplitude fluctuations at the expenses of a poor background noise reduction. In [43], a NOLM setup incorporating an additional phase shifter for extension of the low transmission region of the output characteristic was studied. In the case of a RZ-OOK pulse train, the noise and radiative background in the zerobit time slots is suppressed by the loop mirror saturable absorption action, and the amplitude jitter in the one-bit pulses is also reduced [31-36]. But the NOLM is insensitive to pulse position in time and, thus, it does not aid problems of timing jitter in a system. Nevertheless, its intensity filtering action enables the use of strong guiding filters in the system [31-36] without encountering pulse instability. Fig. 1 (right) shows exemplary optical eye-diagrams of a $40-\mathrm{Gb} / \mathrm{s}$ RZ-OOK signal at the NOLM input and output. Amplitude noise in the input signal is modeled by a Gaussian distribution of the power. It is seen that, although the loss or gain-imbalanced NOLM mainly supports the zero bits, there is amplitude jitter reduction on the one bits too.

\subsection{Ultralong-distance 40-Gb/s transmission}

Efficient growth of the capacity of digital communication systems can be achieved by an increase in the channel bit rate. Increasing the channel rate assumes the utilization of shorter carrier pulses. It is well known that the propagation of ultrashort pulses is strongly affected by the fiber dispersion, which results in large temporal broadening of the carrier pulses. Because of the temporal broadening during propagation, the carrier pulse power spreads over many time slots and so the accumulated effect of the instantaneous fiber nonlinearity tends to get averaged out. Signal transmission using very short optical pulses is often referred to as the quasi-linear regime [86-89]. In this regime, the nonlinear impairments that are allowed to accumulate by the remaining order of the field can impose severe limits to transmission. It is widely accepted that intrachannel nonlinear effects are the dominant sources of nonlinear penalties in quasi-linear fiber communication systems using the conventional OOK (or amplitude-shift keying) modulation format at high bit rates: $40 \mathrm{~Gb} / \mathrm{s}$ or above [86-89]. Intrachannel FWM is responsible for the growth of so-called ghost pulses in the zero-bit time slots and amplitude fluctuations in the one-bit pulses, while intrachannel cross-phase modulation (XPM) primarily causes timing jitter. For the RZ format, transmission in conventional single-mode fiber (SMF) suffers primarily from intrachannel FWM, while it is limited by intrachannel XPM in the TrueWave-class of fibers $[86,87]$. Alloptical $2 \mathrm{R}$ regeneration that suppresses the buildup of a low-power background and reduces the amplitude jitter of the carrier pulses is a possible technique for improving the performance of such systems [11,12,30-36,39]. When a system performance is mainly degraded by amplitude noise and waveform distortion, 
virtually unlimited transmission distances are achievable [31-33,36].

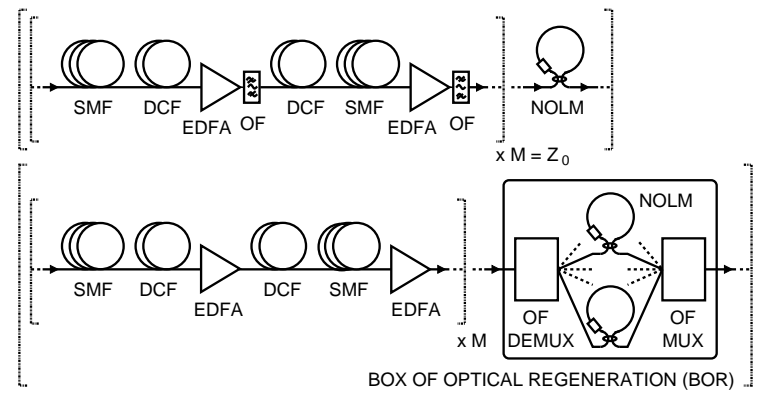

Fig. 2. Unit cell of a periodic transmission line with NOLM-based regenerators: top, single-channel transmission scheme; bottom, WDM transmission scheme.

Figure 2 shows a DM SMF transmission link with in-line NOLM-based 2R regenerators. The transmission link is based on a two-step dispersion map that is composed of SMF spans and dispersion-compensating fiber (DCF) spans. A NOLM is placed into the line every $M$ dispersion map periods (insertion period $Z_{0}$ ). Both single-channel and wavelength-division multiplexed (WDM) transmission schemes include linear control by guiding optical filters (OFs). In the single-channel scheme, filtering can be applied in a combination with each in-line erbium-doped fiber amplifier (EDFA) [31-34] or, more conveniently, a single, equivalent OF can be placed after the amplifier prior to the NOLM location. As we will describe in the following of this section, in the WDM scheme OFs are used to perform signal demultiplexing and multiplexing at each NOLM location $[35,36]$.

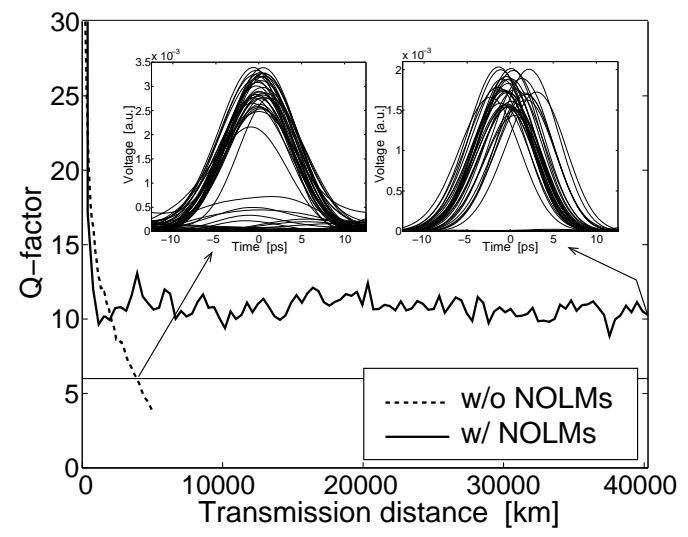

Fig. 3. $Q$-factor versus distance for $40-\mathrm{Gb} / \mathrm{s}$ single-channel RZ-OOK transmission; inset, electrical signal eye-diagrams.

An example of the transmission performance of a system using RZ-OOK single-channel signals at $40-\mathrm{Gb} / \mathrm{s}$ bit rate is given in Fig. 3, which shows the evolution of the conventional (Gaussian-based) $Q$-factor of the received electrical signal over $40,000 \mathrm{~km}$ transmission distance [33]. Note that the $Q$-factor is used here and throughout the paper as a measure of the signal quality rather 

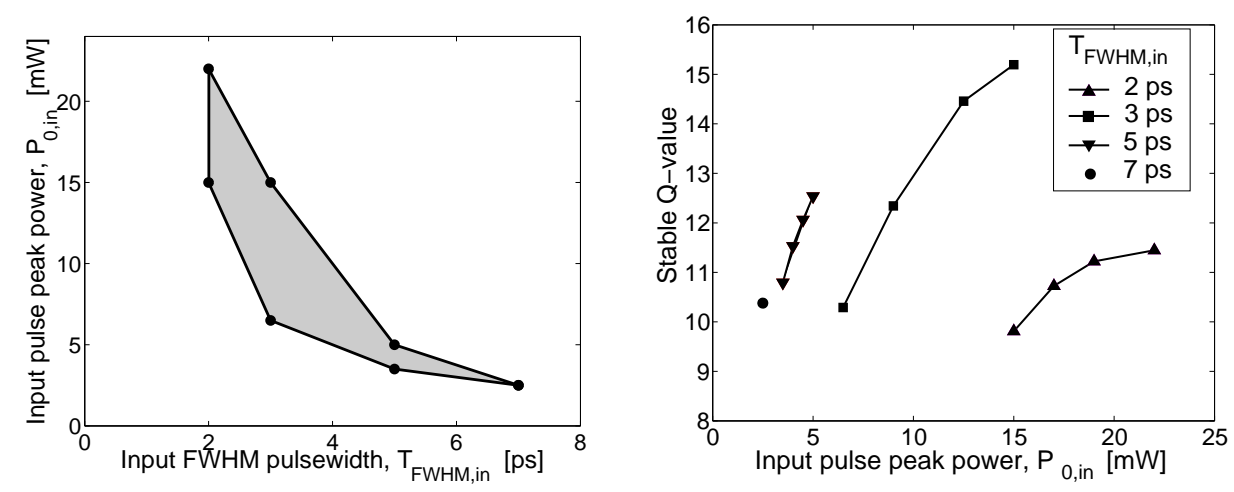

Fig. 4. Working parameter region for distance-unlimited transmission: left, limits of system operation in the plane pulse width-peak power; right, mean value of the stable $Q$ versus input pulse peak power.

than a measure of the bit-error rate (BER). Indeed, in systems employing optical devices with an inherently nonlinear response, the probability density functions of the marks and spaces usually have essentially non-Gaussian shapes and, thus, predictions of the BER based on the $Q$-factor method can yield incorrect results [90]. In the example of Fig. $3, M=5\left(Z_{0}=391 \mathrm{~km}\right)$. We refer to [31-33] for details of the system and numerical modeling. It is seen, from Fig. 3, that for the system without NOLMs, the $Q$-factor decreases and drops below six, set as a limit, after approximately $3,900 \mathrm{~km}$. The result achieved with NOLMs demonstrates that stable transmission over practically unlimited distances at $40 \mathrm{~Gb} / \mathrm{s}$ is possible, where unlimited transmission means here that after some stage, the accumulation of transmission impairments through the system is stabilized, yielding nondecaying $Q$-factor greater than six. Exemplary eye-diagrams of the electrical signal are shown in the inset of Fig. 3. For the system without NOLMs, the eye-diagram indicates that the quality of the transmission is dramatically affected by amplitude noise caused by the intrachannel FWM. It is seen from the eye-diagram in the system with NOLMs that the background noise in the zero time slots is almost completely suppressed by the action of the NOLMs, and the amplitude jitter of ones is also slightly reduced. The eye-diagram reveals that the system will eventually be dominated by timing jitter, but still at $40,000 \mathrm{~km}$ the accumulation of timing jitter is not enough to produce degradation of the $Q$-factor. Figure 4 shows the tolerance limits of distance-unlimited transmission to the parameters of the input pulses for the system of Fig. 3 [33]. The mean value around which the stable $Q$-factor fluctuates against the input pulse peak power for different input full-width at half-maximum (FWHM) pulse widths is also plotted.

Regeneration of WDM signals requires the use of one standard (couplerimbalanced or loss/gain-imbalanced) NOLM for each wavelength channel in order to avoid XPM-induced wave distortion inside the loop mirror. Multichannel $2 \mathrm{R}$ regeneration based on the dispersion-imbalanced loop mirror has been investigated and demonstrated in $[45,46]$. Signal demultiplexing and multiplex- 
ing, before and after the standard NOLM, respectively, can be accomplished with OFs $[35,36]$. Appropriate optical filtering is a promising technique that enables substantial advance in improving the performance of WDM systems [91]. In [36], we have proposed the use of specially designed guiding OFs coupled with NOLMs in a WDM transmission environment. The proposed filter amplitude transfer function is given by

$$
g(\nu)=\exp \left[-\left(\frac{\nu-\nu_{k}}{\Delta \nu^{\prime}}\right)^{2 m}-\left(\frac{\nu-\nu_{k}}{\Delta \nu^{\prime \prime}}\right)^{2}\right], \quad m>1,
$$

where $\nu$ is the optical frequency and $k$ is the channel number. Such a filter function is designed to provide channel separation at the edges and guiding in the center simultaneously. Indeed, the super-Gaussian part of the function provides for sharp edges, while the Gaussian part allows for a guiding top. Parameter $m$ controls the degree of edge sharpness in (2). This filter function can be easily implemented with existing technologies. For instance, one may use a fiber Bragg grating with the prescribed apodisation profile. We remark that, unlike filters that are commonly placed into a transmission line to limit the bandwidth of the noise, the WDM filters considered here do affect pulse propagation. This implies that good system performance depends on the proper balance between the filter edge-cutting and guiding effects.
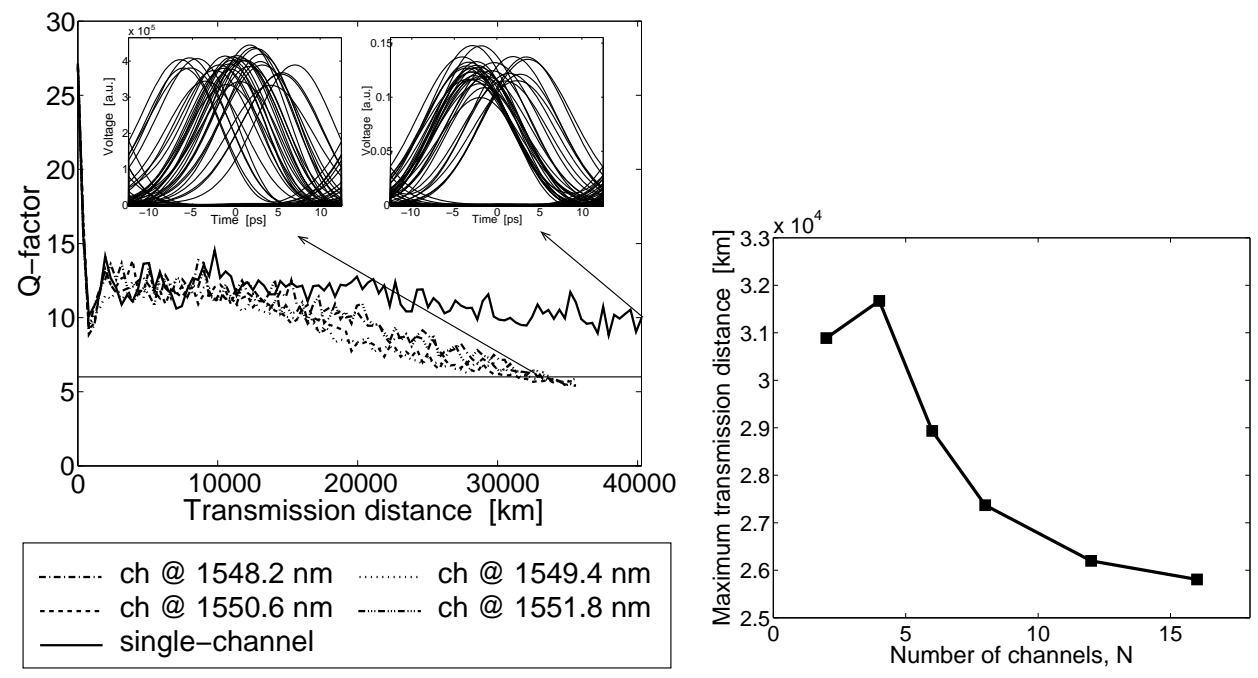

Fig. 5. Left, $Q$-factor versus distance for $40-\mathrm{Gb} / \mathrm{s} \times 4150 \mathrm{GHz}$-spaced $\mathrm{WDM}$ and 40-Gb/s single-channel RZ-OOK transmissions; inset, electrical signal eye-diagrams. Right, maximum transmission distance of the worst channel versus number of channels.

Figure 5 (left) shows an example of the transmission performance of a WDM RZ-OOK system at $40-\mathrm{Gb} / \mathrm{s}$ per-channel bit rate, where the regeneration method based on the WDM filters specified by (2) and NOLMs is applied [36]. In this example, a DM SMF transmission link similar to that of [3133 is modeled, and four wavelength channels spaced by $150 \mathrm{GHz}$ are used. 
We refer to [36] for system and modeling details. A transmission distance of more than $30,000 \mathrm{~km}$ with a $Q$-factor greater than six is reached by all channels in the example of Fig. 5. On the other hand, single-channel transmission over practically unlimited distances is possible in the WDM system. This result corresponds to the result reported in Fig. 3, and indicates that the WDM transmission performance is only affected by interchannel effects. This is evident from the electrical signal eye-diagrams in the inset of Fig. 5 (left). The WDM channel eye-diagram shows that the WDM performance is mainly degraded by timing jitter induced by the interchannel XPM. The single-channel eye-diagram is still open at $40,000 \mathrm{~km}$, showing that the accumulation of intrachannel timing jitter through the system is not enough at this stage to produce significant degradation of the $Q$-factor. In both WDM and single-channel transmissions, in fact, intrachannel FWM is not a limiting factor owing to the loop mirror intensity filtering action. The sensitivity of the proposed regeneration technique to the number of transmitted wavelength channels is shown in Fig. 5 (right), where the maximum transmission distance for the worst channel is plotted as a function of the number of channels [36].

We point out that in 40-Gb/s strongly DM RZ-OOK transmission systems that are dominated by both intrachannel FWM and XPM, the application of the NOLM-based $2 \mathrm{R}$ regeneration technique still can dramatically improve the system performance, allowing for transmission over transoceanic distances [34].

\subsection{Application in 40-Gb/s all-optical networks}

Most of the research on all-optical signal regeneration has focused up to now on characterization and design optimization of the optical regenerators (ORs) and their deployment in the elite ultralong communication systems. In such systems, the function of periodically installed in-line ORs is to recover the optical signals in order to achieve the longest possible error-free transmission. Networking applications of ORs have not as extensively studied. In the terrestrial networks, where the typical distance between nodes is shorter than a thousand kilometers, ORs can be located directly at the nodes and used to improve the optical signal-to-noise ratio and the signal quality before the next stage. There are several technical solutions that can be applied to realize $2 \mathrm{R}$ and $3 \mathrm{R}$ signal regeneration within a terrestrial network. From a practical viewpoint, however, performance improvement should be combined with low implementation cost. With transmission distances less than a thousand kilometers, simple and cost-efficient $2 \mathrm{R}$ regenerative devices can be an attractive practical solution.

In $[37,38]$, have analyzed the performance of the NOLM-based $2 \mathrm{R}$ regenera- 

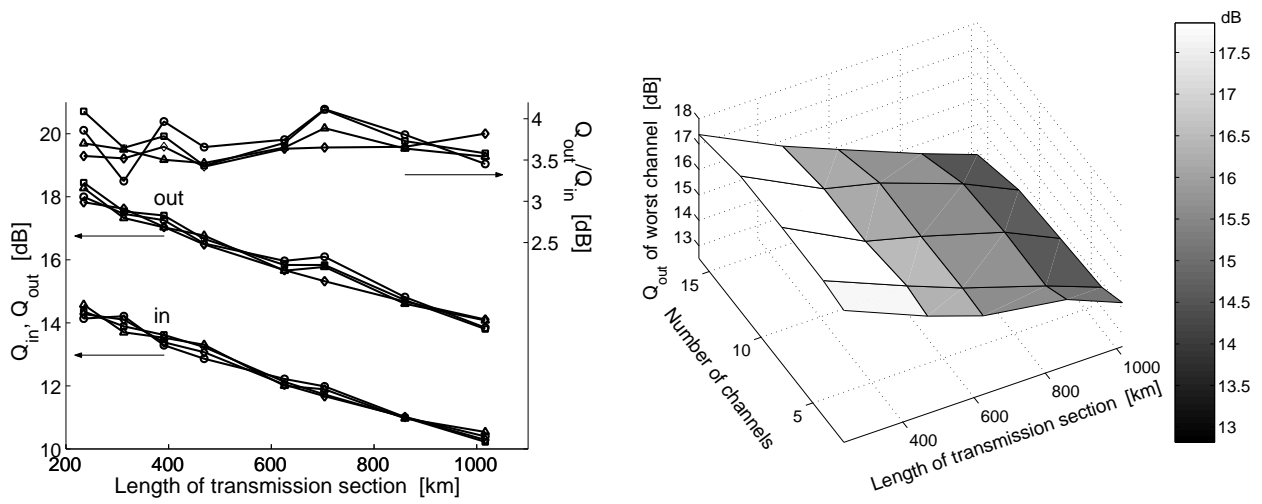

Fig. 6. Left, $Q$-value at the regenerator input and output and improvement factor versus length of the transmission section for $40-\mathrm{Gb} / \mathrm{s} \times 4150 \mathrm{GHz}-\mathrm{spaced}$ WDM RZ-OOK signal. Right, regenerated $Q$-value of the worst channel versus transmission span length and number of channels.

tor in SMF-based optical networks operated at 40-Gb/s per-channel bit rate. The regeneration scheme used in $[37,38]$ consisted of a DM SMF transmission section followed by a box of optical regeneration (BOR), similar to the unit cell of the periodic transmission system depicted in Fig. 2. Fig. 6 (left) shows an example of the regenerator performance as a function of the length of the transmission section for a four-channel WDM signal with $150 \mathrm{GHz}$ channel spacing [37]. The remarkable observation from this figure is that the rate of variation of the regenerated $Q$-factor, $Q_{\text {out }}$, with the transmission length is approximately equal to that of the $Q$-value at the regenerator input, $Q_{\text {in }}$, leading to a similar improvement factor $Q_{\text {out }} / Q_{\text {in }}$ (between 3.2 and $4.1 \mathrm{~dB}$ in this example) for all the channels at any of the distances considered. This result demonstrates the ability of the proposed regeneration scheme to satisfy the demand for network scalability. In Fig. 6 (right), the dependencies of the $Q$-factor of the worst channel at the regenerator output on the transmission span length and the number of channels are plotted [37]. One may see that the quality of the regenerated signals is almost independent of the number of channels, which indicates the potential of the scheme to be used in high-capacity WDM networks.

We point out that the regeneration of the pulse amplitude and shape performed by the NOLM-based regenerator is independent of the fiber link and resides solely in the loop mirror intensity filtering action. In principle, this makes the regenerator an ideally black box of pulse reamplification and reshaping suitable for different fiber systems, thus providing for network reconfigurability. Obviously, as the NOLM cannot directly improve the timing jitter in a system, other methods should be employed to improve performance in this respect. A comparison of the performances of the NOLM-based $2 \mathrm{R}$ regenerator and a 3R regenerator employing a synchronous amplitude modulator (AM) enhanced by HNLF [26] showed that the simple NOLM-based OR performs 
very well (and sometimes even better than the more complex 3R OR) when applied after transmission spans of a thousand kilometers, while the AM-based OR is more efficient in longer connections when the signals suffer from severe degradation [38]. Evidently, the outcome of the comparison of ORs depends on various conditions such as the nature of signal impairments to be restored and requirements for efficiency and tolerance. As mentioned previously, the cost issue is also an important parameter of comparison. The results obtained in [38] indicated that a combination of $2 \mathrm{R}$ regeneration at each node and full $3 \mathrm{R}$ regeneration at some critical points where high regeneration performance is of crucial importance could be an attractive design of a flexible all-optical network.

\section{Autosolitons in fiber lines with lumped nonlinear optical devices}

As far as the theory is concerned, in-line optical signal processing is a reach area of research for general nonlinear science and, in particular, for soliton theory. Self-organization phenomena in optical fiber systems based on the fiber nonlinearity and dispersion and the nonlinear effects introduced by in-line dissipative optical devices can be utilized to produce autosolitons. The term "autosolitons" or "dissipative solitons" here and throughout the paper means robust localized pulses, with parameters prescribed by properties of the system, which often occur in nonlinear dissipative models [72,92-95]. Fiber transmission systems exploiting NODs can take advantage of both a self-supporting, stable carrier signal and the signal regeneration function performed by such devices. However, because of the complexity of such nonlinear systems, typically, extensive numerical modeling is required to identify promising operational regimes. The quasi-linear operating regime of high-speed transmission systems is, in some sense, the opposite of soliton [96] or DM soliton [97] transmission, where the fiber nonlinearity plays an important role in preserving the pulse shapes during propagation. In this regime, the in-line Kerr nonlinearity is almost a "negative" factor contributing to the destabilization and distortion of signal pulses. Therefore, a certain amount of "constructive" nonlinearity is required to stabilize ultrashort pulse propagation and, thus, to improve the system performance. We have numerically demonstrated in [30-33] that, under certain conditions, the interplay between fiber dispersion, the lumped nonlinearity provided by in-line NOLMs, and the action of linear control elements, such as OFs, leads to the formation of autosolitons. These are periodically reproduced at the output of each segment of the transmission line. The use of autosolitons as carrier signal pulses was the key for success of the stable signal transmission over ultralong distances described in Section 2. Here, we outline the key points of a theory of optical signal transmission in DM fiber systems in the quasi-linear regime, with periodically placed in-line NODs [78,79]. Ap- 
plication of this general theory to the specific example of a system with in-line NOLMs is demonstrated.

\subsection{The mapping problem}

Following [78,79], we consider the optical pulse propagation in a cascaded transmission system with periodic variations of dispersion and nonlinearity, optical amplification, frequency filtering, and NOD management. To simplify the full nonlinear Schrödinger equation (NLSE) model, we make some justified physical assumptions. We analyze the case of linear propagation in fiber, when the nonlinear term in the NLSE can be neglected. Such a propagation regime corresponds to the case where the nonlinear length in the fiber is much longer than the local dispersion length. The transformation of a pulse after propagation in one segment of the periodic transmission line can be considered as the mapping of an input pulse into an output one. Considering an element of the transmission line that includes a NOD specified by its power-dependent amplitude transfer function $f(P)$, a piece of linear fiber of length $Z_{0}$, and $m$ filters, the mapping of the signal, defined up to a phase factor $\mu$, can be represented by $[78,79]$

$$
e^{i \mu} U_{n+1}(t)=\int_{-\infty}^{+\infty} \mathrm{d} t^{\prime} K\left(t-t^{\prime} ; Z_{0}\right) f\left(\left|U_{n}\left(t^{\prime}\right)\right|^{2}\right) U_{n}\left(t^{\prime}\right), \quad n=0,1, \ldots
$$

Equation (3) is one of the central results of the theory presented in this section. This mapping problem plays a fundamental role in the description of highbit-rate fiber communication systems. In Eq. (3), $U(z, t)=Q^{-1}(z) u(z, t)$, where $u(z, t)$ is the slowly-varying pulse envelope in the comoving system of coordinates, $Q(z)=\exp \left[-\int_{(k-1) Z_{a}}^{z} \mathrm{~d} z^{\prime} \gamma\left(z^{\prime}\right)\right]$ for $(k-1) Z_{a}<z<k Z_{a}$, and $Q(z)=1$ for $z=k Z_{a}^{+}$. Here, $Z_{a}=Z_{0} / p$ is the amplifier insertion period with $p$ an integer number, and $\gamma(z)$ is the fiber loss coefficient that accounts for the signal attenuation along the fiber span before the $k$ th amplifier. The NOD is assumed to be placed immediately after an amplifier, and the signal is taken at the input point $n Z_{0}^{-}$to the NOD after any device prior to the NOD. The kernel $K$ describes signal propagation in the unit cell $Z_{0}$. For instance, in the case when Gaussian filters are used, $K$ can be written in the form $[78,79]$

$$
K\left(t-t^{\prime} ; Z_{0}\right)=\sqrt{G} \sqrt{\frac{i}{2 \pi\left(B_{0}+i m / \Omega_{f}^{2}\right)}} \exp \left[-\frac{i\left(t-t^{\prime}\right)^{2}}{2\left(B_{0}+i m / \Omega_{f}^{2}\right)}\right] .
$$

In Eq. (4), $\Omega_{f}$ is the filter bandwidth, $B_{0}=\int_{n Z_{0}}^{(n+1) Z_{0}} \mathrm{~d} z \beta_{2}(z)$, where $\beta_{2}(z)$ is the varying fiber group-velocity dispersion (GVD), and $G$ is an excess gain that accounts for compensation of the signal energy losses introduced by the NODs and filters in the system. From a transmission point of view, it is desirable to 
find (if it exists) a steady-state propagation regime in which an optical pulse propagating along the transmission line reproduces itself periodically at the output of each element of the line. That corresponds to determining a fixed point of the mapping (3). Therefore, in order to find the steady-state pulse shape $U(t)$, one has to solve the nonlinear integral equation which stems from (3) if we put $U_{n+1}(t)=U_{n}(t)=U(t)$. If the steady-state pulse is stable, then any initial signal within the basin of attraction of the fixed point will gradually evolve towards it after some mapping intervals. Henceforth, we will focus on the case when the NODs are NOLMs specified by (1).

\subsection{Dissipative soliton solutions and approximate mapping}

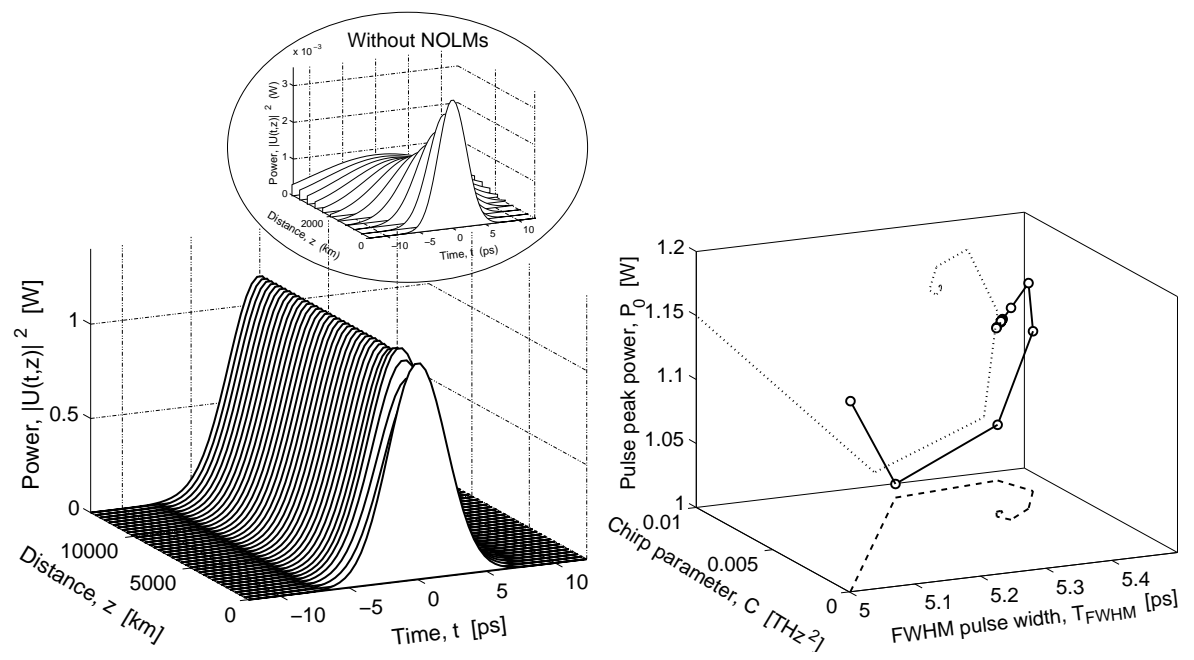

Fig. 7. Stroboscopic pulse evolution as viewed at the NOLM input: left, evolution of the intensity profile; inset, intensity profile evolution in the system without NOLMs; right, acquisition of the steady state in the space pulse width-chirp parameter-peak power.

First, we demonstrate the feasibility of stable dissipative soliton propagation guided by in-line NOLMs, by direct numerical simulations of the basic NLSE propagation model. The sample transmission scheme used in the numerical integration of the NLSE is similar to that of [31-33] (see Fig. 2) except that a single $(m=1)$ OF is placed after the amplifier prior to the NOLM location. We note that fiber nonlinearity is included in the calculations. We refer to $[78,79]$ for system and modeling details. Figure 7 shows an example of pulse evolution in the system, measured stroboscopically at the NOLM input point $[78,79]$. This is the key demonstration of the dissipative nature of the considered nonlinear structures. In contrast to conservative Hamiltonian solitary waves, where the pulse power is a continuous function of the width, here the dissipative soliton parameters are fixed by the system characteristics. The solution is an attractor that provides robustness and stability of these signal pulses. One may see, from Fig. 7, that the pulse settles down to a steady state 
after a short initial transient, in contrast to the case without NOLMs, where the pulse peak power decays and the pulse width broadens with distance. This result demonstrates the feasibility of stable pulse propagation in the system, and indicates that the use of in-line NOLMs converts the quasi-linear transmission regime into a dissipative soliton transmission regime, which is strictly nonlinear [30-33]. We note that the same stroboscopic picture as that in Fig. 7 can be obtained by simply iterating the mapping equation (3). Figure 8 (left) shows the basin of attraction of the steady-state solution of Fig. 7 in the plane FWHM pulse width vs. chirp parameter [78,79]. It is seen that there is a large tolerance to the initial pulse width and chirp, which indicates a high degree of stability of the steady-state solution. The tolerance limits of stable pulse propagation to the filter bandwidth and the path-averaged dispersion of the line are shown in Fig. 8 (right). There, $\delta \nu_{f}=\sqrt{\ln 2} \Omega_{f} / \pi$, and $\langle D\rangle=-2 \pi c_{0}\left\langle\beta_{2}\right\rangle / \lambda^{2}$, where $\lambda$ is the carrier wavelength, $c_{0}$ is the speed of light, and $\langle\cdot\rangle$ denotes the average over a dispersion compensating period. Figure 8 (right) illustrates how the system parameters can be used to select desirable stable regimes with a particular average dispersion. Note that this is a feature that is not always possible in conservative fiber transmission systems. Specifically, it means that the use of dissipative solitons as information carriers provides more freedom and opportunities in the design of transmission systems.
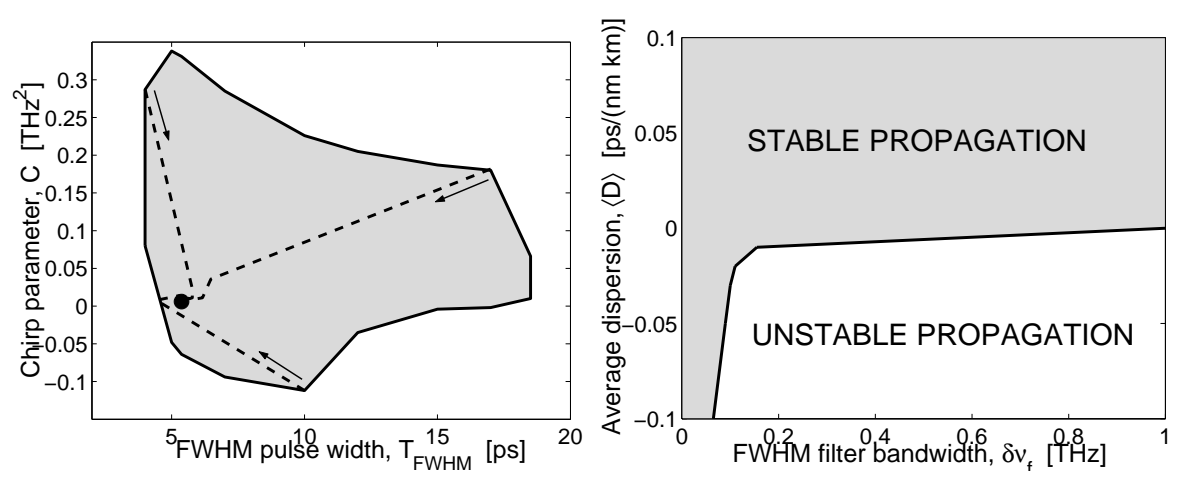

Fig. 8. Left, basin of attraction of the steady-state solution of Fig. 7 in the plane pulse width-chirp parameter. Right, limits of stable pulse propagation in the plane filter bandwidth-average dispersion.

As we pointed out previously, in order to find a steady-state pulse propagation regime, one has to solve a mapping integral equation (3) for the fixed points. While this can be done numerically, it is very useful to have a simplified approximate method to find solutions of the basic model for massive optimization of the system parameters. Following $[78,79]$, here we apply a simple variational approach to describe the characteristics of the dissipative soliton solutions found above. We notice, from full numerics, that the steady-state pulse shape at the NOLM input point can always be fitted well by a Gaussian profile. Thus, we choose a trial input pulse $U(t)$ for the map as a Gaussian-shaped pulse with (yet unknown) peak power $P_{0}$, root-mean-square (RMS) width $T_{\mathrm{RMS}}$, and RMS 
chirp parameter $C_{\mathrm{RMS}}: U_{n}(t)=\sqrt{P_{0}} \exp \left[-t^{2} /\left(4 T_{\mathrm{RMS}}^{2}\right)+i C_{\mathrm{RMS}} t^{2}\right]$. The output of the map $U_{n+1}(t)$ given by Eq. (3) will be non-Gaussian in general, but it will have a similar shape and will depend on the parameters of the input signal. Let us now demand that the peak power, pulse width, and chirp of the output signal coincide with those of the input Gaussian signal. This provides a system of transcendental equations for the sought parameters $P_{0}, T_{\mathrm{RMS}}$, and $C_{\mathrm{RMS}}$,

$$
\begin{gathered}
P_{0}=\frac{1}{\sqrt{2 \pi} T_{\mathrm{RMS}}} \int_{-\infty}^{+\infty} \mathrm{d} t\left|U_{n+1}\left(t ; P_{0}, T_{\mathrm{RMS}}, C_{\mathrm{RMS}}\right)\right|^{2}, \\
T_{\mathrm{RMS}}^{2}=\frac{\int_{-\infty}^{+\infty} \mathrm{d} t t^{2}\left|U_{n+1}\left(t ; P_{0}, T_{\mathrm{RMS}}, C_{\mathrm{RMS}}\right)\right|^{2}}{\int_{-\infty}^{+\infty} \mathrm{d} t\left|U_{n+1}\left(t ; P_{0}, T_{\mathrm{RMS}}, C_{\mathrm{RMS}}\right)\right|^{2}} \\
C_{\mathrm{RMS}}=\frac{\operatorname{Im} \int_{-\infty}^{+\infty} \mathrm{d} t U_{n+1}^{2}\left(t ; P_{0}, T_{\mathrm{RMS}}, C_{\mathrm{RMS}}\right)\left(\partial_{t} U_{n+1}^{*}\left(t ; P_{0}, T_{\mathrm{RMS}}, C_{\mathrm{RMS}}\right)\right)^{2}}{\int_{-\infty}^{+\infty} \mathrm{d} t\left|U_{n+1}\left(t ; P_{0}, T_{\mathrm{RMS}}, C_{\mathrm{RMS}}\right)\right|^{4}} .
\end{gathered}
$$

If the solution of system (5) exists, then it provides a variational approximation for the parameters of the steady-state pulse $[78,79]$. In particular, one may use the Gaussian ansatz with the found vaues of $P_{0}, T_{\mathrm{RMS}}$, and $C_{\mathrm{RMS}}$ as an approximation of the steady-state pulse shape. An example of the comparison between the theoretical predictions from the variational model (Eqs. (5)) and the results of full numerical simulations is given in Fig. 9, where the steadystate RMS pulse width and chirp parameter are plotted as functions of the filter bandwidth [78,79]. The solutions of Eq. (3) are also plotted. It is seen that the theory agrees well with the full numerics.

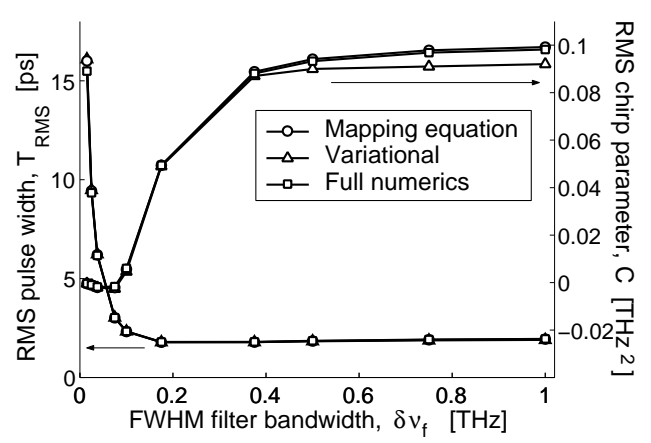

Fig. 9. Steady-state RMS pulse width (left axis) and chirp parameter (right axis) at the NOLM input point versus filter bandwidth. 


\section{All-optical nonlinear signal processing at an optical receiver and in-line format conversion}

The full potential of all-optical data processing is still to be realized in a variety of possible applications. An attractive approach to the design of the optical receiver for high-speed optical fiber communication systems using RZ data formats is based on employing an additional nonlinear all-optical decision element (ODE) just before the conventional receiver [21,23,82,98-103]. In this way, the first decision is carried out in the optical domain, thus improving the quality of the signal which is received. In [98], the use of Kerr nonlinearity in a NDF has been introduced as a technique to reduce the impact of timing jitter at a RZ receiver. In this section, we describe a novel, simple scheme of all-optical nonlinear pulse processing that combines the nonlinear temporal pulse broadening in a NDF for improvement of the phase margin of the optical signals with the intensity filtering action of a NOLM for signal reamplification and cleaning up [80-82]. We also demonstrate that such pulse processors permit to realize optical data transmission with periodic in-line all-optical conversion between RZ and NRZ-like modulation formats [83].

\subsection{Configuration, principle and operation of the pulse processor}

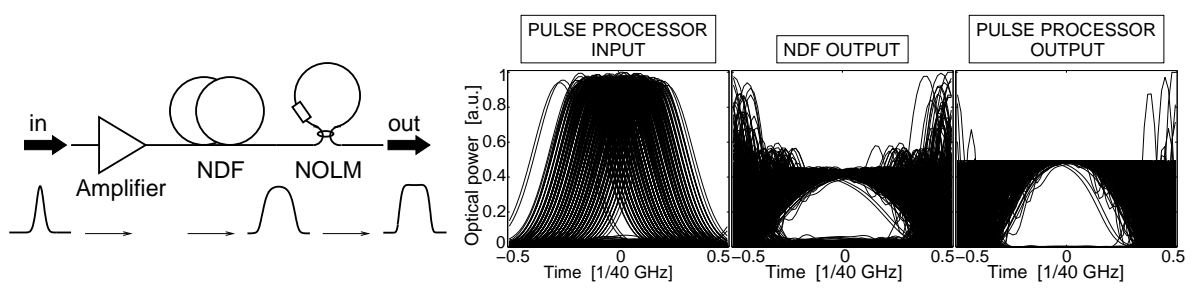

Fig. 10. Left, scheme of the pulse processor. Right, optical signal eye-diagrams in the pulse processor.

The proposed pulse processor consists of an optical amplifier, a section of NDF, and an imbalanced NOLM (Fig. 10). Qualitatively, the idea for the method is as follows [80,81]: an input optical pulse to the pulse processor is amplified to the preferred power level of the device by the amplifier. During transmission along the NDF, the temporal waveform of the pulse is changed to a rectangular-like profile by the combined action of GVD and Kerr nonlinearity [104]. Note that, if we use distributed amplification in the NDF instead of lumped amplification at the pulse processor input, then the pulse temporal waveform will be changed to a parabolic profile by dispersion, nonlinearity and gain [81]. After propagation in the NDF, the pulse width is broadened and the central portion of the pulse changes to become flat. The phase margin of a RZ pulse train is improved by utilizing this property [98]. This in turn reduces the influence of the displacement of pulse position in time caused by 
timing jitter. Indeed, broadening of the pulse width to approximately a 1 bit duration causes the center-of-mass of the pulse portion contained in the bit slot to move towards the top of the pulse, where timing jitter is less than in the tails as a result of flattening of the pulse envelope. In [102], we have demonstrated that the temporal pulse broadening and flattening in a NDF can be utilized to improve the BER of a signal in an ODE. Following the NDF, the pulse enters the NOLM. As we have described in Section 2, the imbalanced NOLM offers pulse reamplification and cleaning up through its intensity filtering action, and pulse amplitude stabilization for operation just after the peak of its switching curve. This reduces the amplitude noise of a RZ pulse train. Note the proposed pulse processor acts as an all-optical waveform converter.
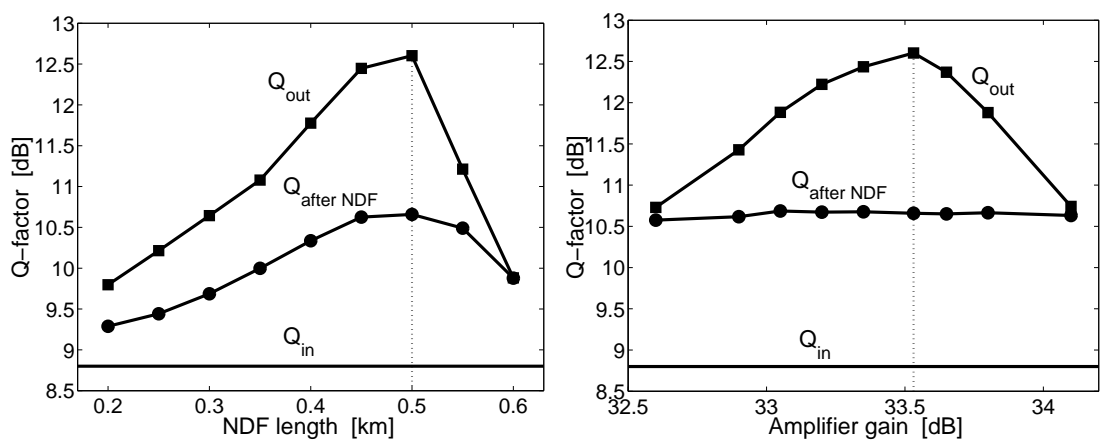

Fig. 11. $Q$-factors in the pulse processor versus length of the NDF (left) and gain of the amplifier (right).

An example of the pulse processor action on RZ signals is given by the optical signal eye-diagrams in Fig. 10 (right). Timing jitter and amplitude noise in the $40-\mathrm{Gb} / \mathrm{s}$ input signal are modeled by Gaussian distributions of the pulse temporal position and signal power, respectively. The eye of the input signal closes mainly because of significant timing jitter with the pulses. When the proposed pulse processing scheme is employed, the broadening of the pulse width and simultaneous flattening of the pulse shape in the NDF yields an increase in eye opening. The amplitude jitter of the pulses at the center of the bit slot is also slightly smaller after propagation in the NDF. The additional widening of eye opening that can be observed at the pulse processor output is given by a significant reduction of the amplitude noise in both zero and one bits provided by the NOLM. The tolerance of the pulse processor performance to intrinsic device parameters for input timing-jitter degraded $40-\mathrm{Gb} / \mathrm{s}$ signals is illustrated in Fig. 11 [80,81].

\subsection{All-optical decision element at an optical receiver}

The structure of an ODE-enhanced receiver is shown in Fig. 12. The receiver is constructed from an optical bandpass filter $(\mathrm{OBPF}) /$ demultiplexer, a photode- 


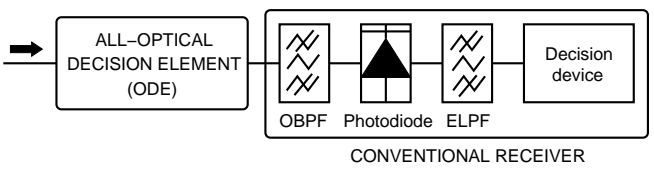

Fig. 12. Scheme of the RZ optical receiver.

tector with a square-law characteristic, an electrical low-pass filter (ELPF), and a threshold detector at the decision point. A nonlinear ODE is placed in front of the receiver. Henceforth, we will focus on the case when the ODE is the NDF-enhanced NOLM described above. The OBPF filters the incoming optical signal, removing the accumulated out-of band amplifed spontaneous emission (ASE) noise generated by in-line amplifier repeaters at the detector. The ELPF shapes the voltage signal. It has two functions. One is that the filter limits the receiver bandwidth in order to further reduce the noise both accumulated during the transmission stage and generated in the receiver. The other one is that the filter expands the pulse width provided that the duty ratio of the received pulse is sufficiently small and, therefore, reduces the influence of the fluctuation of the temporal pulse position caused by timing jitter [105]. However, there exists a trade-off in the cutoff frequency of the electrical filter among the effects of intersymbol interference (ISI), signal amplitude reduction, and signal-to-noise ratio (SNR) improvement.
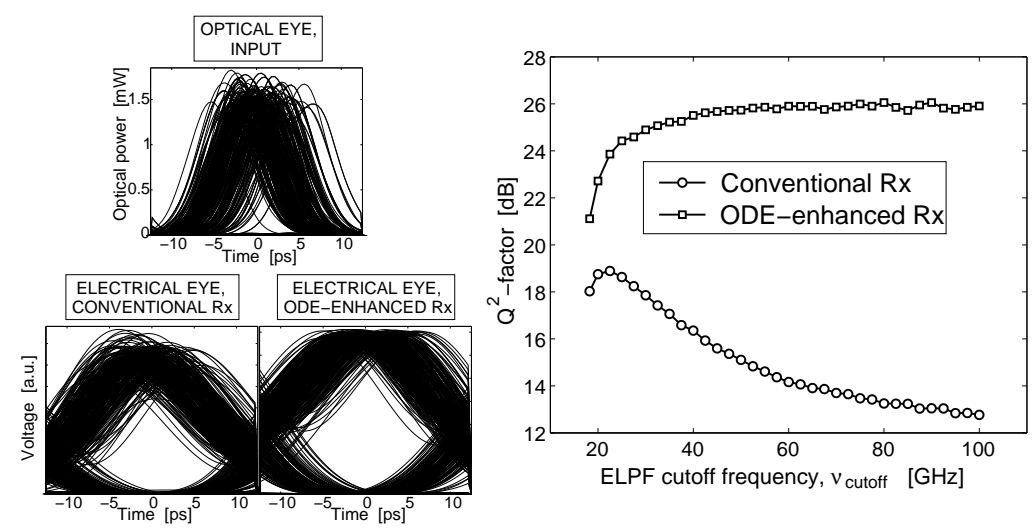

Fig. 13. Receiver performance: left, signal eye-diagrams in the receiver; right, $Q^{2}$-factor versus cutoff frequency of the electrical filter.

The improvement of signal quality that can be achieved in the ODE-modified receiver with respect to the conventional receiver is illustrated in Fig. 13 [82]. Figure 13 (left) shows an example of eye-diagrams of a 40-Gb/s optical signal mainly degraded by timing jitter at the input to the receiver, and of the detected electrical signal in the two receiver types. When the pulses are detected with the conventional scheme, the pulse width is broadened by the ELPF, and this results in increase of the eye opening. The eye opening detected with the proposed scheme is larger. This is due to the simultaneous reduction of timing and amplitude jitters provided by the NDF-enhanced NOLM. In Fig. 13 (left), the signal $Q^{2}$-factor is plotted as a function of the cutoff frequency of the ELPF for the two receiver types. We refer to [82] for details of the system. 
For the conventional receiver, when the cutoff frequency is too low, the signal quality is degraded by the increase of ISI and the decrease of pulse amplitude. Large cutoff frequency leads to a decrease of the SNR in the detected electrical signals, and this also results in signal quality degradation. On the other hand, when the proposed method is employed, the nonlinear pulse broadening in the NDF permits to improve the signal phase margin without increasing the ISI. The nonlinear loop mirror intensity filtering improves further the quality of the detected signals. In the proposed scheme, the required electrical bandwidth is wider than that of the conventional receiver since also the pulse spectrum spreads out. However, because of the nonlinearity involved in the process, the $\mathrm{SNR}$ is not decreased for a wide range of cutoff frequencies. Indeed, it is seen that the $Q$-factor stabilizes and does not degrade any further after some value of the cutoff frequency in the considered frequency range. We mention that for larger cutoff frequencies the signal quality will be eventually degraded by the receiver thermal noise.

\subsection{Data transmission with periodic in-line all-optical format conversion}

In conventional transmission lines operated at high bit rates, for both RZ and NRZ formats transmission is limited by the accumulation of nonlinear impairments along the line [87,106-108]. In long-haul transmission systems RZ turns out to be superior compared to RZ [109], in virtue of a higher robustness to nonlinear signal distortion. To reduce the impact of nonlinearity, typically NRZ is used at low powers, and consequently, systems employing such a modulation format are mainly limited by degradation of the SNR due to accumulation of ASE noise. In high bit-rate, strongly DM RZ systems, the optimal transmission regimes are at higher powers compared to NRZ and, as previously mentioned, are limited by intrachannel nonlinear effects. Even when the signal amplitude noise is reduced [31-34,110-112], timing jitter can be still an important limiting factor in such systems. It is also well-known that the NRZ format is resistant to timing jitter. Thus, in high bit-rate systems with strong dispersion management the key limiting factors are different for different data formats or, in other words, are format dependent. All-optical modulation format conversion might become a necessary technology for future all-optical networks, which may employ miscellaneous formats. Various alloptical format converters between RZ and NRZ including NOLMs [113] and SOAs $[114,115]$ have been demonstrated in back-to-back schemes. Here, we describe a novel transmission technique based on periodic in-line all-optical format conversion between RZ and NRZ-like [83]. The aim of the approach is to alternate format-specific transmission impairments in order to delay their accumulation along the link. As a particular realization of the general idea, we consider a system with in-line nonlinear signal processors based on NDFenhanced NOLMs at 40-Gb/s data rate. 


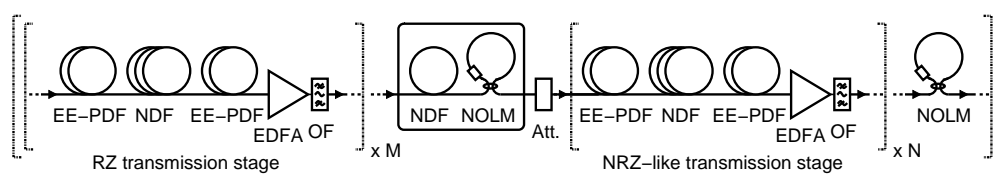

Fig. 14. One unit cell of the periodic transmission line.

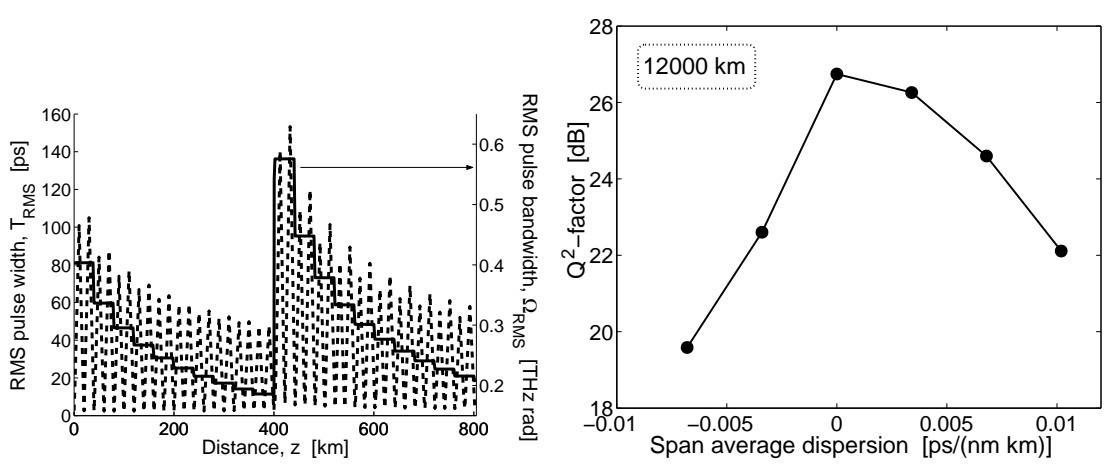

Fig. 15. Left, evolution of the steady-state RMS pulse width and bandwidth over one period of the system. Right, $Q^{2}$-factor at the NDF-NOLM signal processor output versus span average dispersion of the second cell of the system period.

The transmission scheme is depicted in Fig. 14. One element of the periodic transmission line is composed of two cells, which amount to $M$ and $N$ amplifier spans, and are followed by a NDF-NOLM based pulse processor and a conventional NOLM, respectively. Each amplifier span consists of effective core area enlarged positive dispersion fiber (EE-PDF) and NDF. We refer to [83] for details of the system. In the absence of regenerators, the transmission perofrmance of the system is severely degraded by both nonlinear interactions-induced amplitude noise and timing jitter [34]. The RZ pulses transmitted in the first stage are reamplified, cleaned up, and converted to pulses with a NRZ-like waveform by the nonlinear pulse processor. After the RZ-format transmission stage, the timing jitter of pulses is accumulated. An optical attenuator lowers the pulse power at the pulse processor output. In the second stage, the lower-power NRZ-like pulses are gradually reconverted to RZ pulses by the combined action of optical filtering and fiber dispersion, and $2 \mathrm{R}$ regenerated by the NOLM. The partial propagation in the form of NRZ-like reduces the timing jitter accumulation. Figure 15 (left) shows the evolution of the RMS pulse width and bandwidth of a steady-state pulse for the system over one period of the system $(800 \mathrm{~km}, M=N=10)$. [83]. The starting and ending points are the output and input of the conventional NOLM, respectively. One may see that the pulse dynamics along the transmission line is quasi-linear. Indeed, the pulse bandwidth keeps a constant value within each amplifier span, while undergoing a jump at the filter locations. On the other hand, the bandiwtdh exhibits a large change at the locations of the NODs placed in the system. This is an indication of the conversion of the quasilinear propagation regime into a stable autosoliton nonlinear regime by the NODs. Figure 15 (left) illustrates the system performance through evaluation 
of the tolerance of the transmission scheme to the path-averaged dispersion of the line [83]. In this example, the line path-averaged dispersion is tuned by varying the span average dispersion in the second cell of the periodicity element of the system, and the results are shown after 12,000 km transmission. It is seen that the optimum average dispersion is zero. This dispersion value yields unchirped pulses at the nonlinear signal processor output.

\section{All-optical $2 \mathrm{R}$ regeneration of phase-encoded signals}

Phase modulation as a method for information encoding in optical fiber communications has attracted intense interest in recent years. Advantages of using PSK modulation formats include higher receiver sensitivity over conventional OOK systems and suitability for multi-level signaling [116-118]. However, unlike the OOK signals, the PSK signals are sensitive to phase noise. In PSK transmission systems, linear phase noise is imposed on the signal by ASE from OAs. Moreover, in long-distance systems, nonlinear phase noise, which is caused by the translation of amplitude noise to phase noise through the Kerr nonlinearity of the transmission fiber [119], contributes significantly to limiting the system performance [120]. All-optical regeneration of PSK transmission by low-complexity and cost-efficient devices that can handle binary phase information would be an attractive technique to improve the performance of PSK systems. While most of the regenerators studied so far have been designed to regenerate OOK signals without particular attention to restoration or preservation of the signal phase, some recent works aim at realizing phase regenerative or phase transparent signal regeneration [84,121-131]. Although ultimate regeneration of binary phase information may require phasesensitive amplifiers [121,122], regenerators that regulate the signal amplitudes with the phase information preserved (not regenerated) are expected to be useful [84,125-131]. The PSK system performance can still be improved by in-line phase-preserving $2 \mathrm{R}$ regenerators, because the employment of such devices suppresses the accumulation of amplitude noise and waveform distortion through the system and, correspondingly, limits the growth of phase noise [84,129-131]. As we have described in Section 2, all-optical 2R signal regeneration based on the fiber NOLM has shown excellent regenerative properties using OOK signals. However, the major problem when using traditional regeneration schemes based on the NOLM with PSK signals is a strong conversion of the input signal amplitude jitter into phase jitter, since the conventional NOLM has a transfer function with a linear dependence of the output phase on the input signal power (see (1)). In [126,127], a modified NOLM was studied, where directional attenuation enables maintaining the binary phase information, which is inherently degraded by the conventional NOLM. In this section, we present a phase-preserving all-optical $2 \mathrm{R}$ regeneration scheme for 
phase-encoded signals, which is based on a new design of RA-NOLM [84]. We furthermore demonstrate that the combination of the RA-NOLM with narrow spectral filtering can provide an additional phase-limiting function in PSK signal transmission, by suppressing the accumulated ASE-induced phase noise. As an example, we consider a RZ-DPSK system operated at 40-Gb/s data rate.

\subsection{Operation principle and configuration of the RA-NOLM}
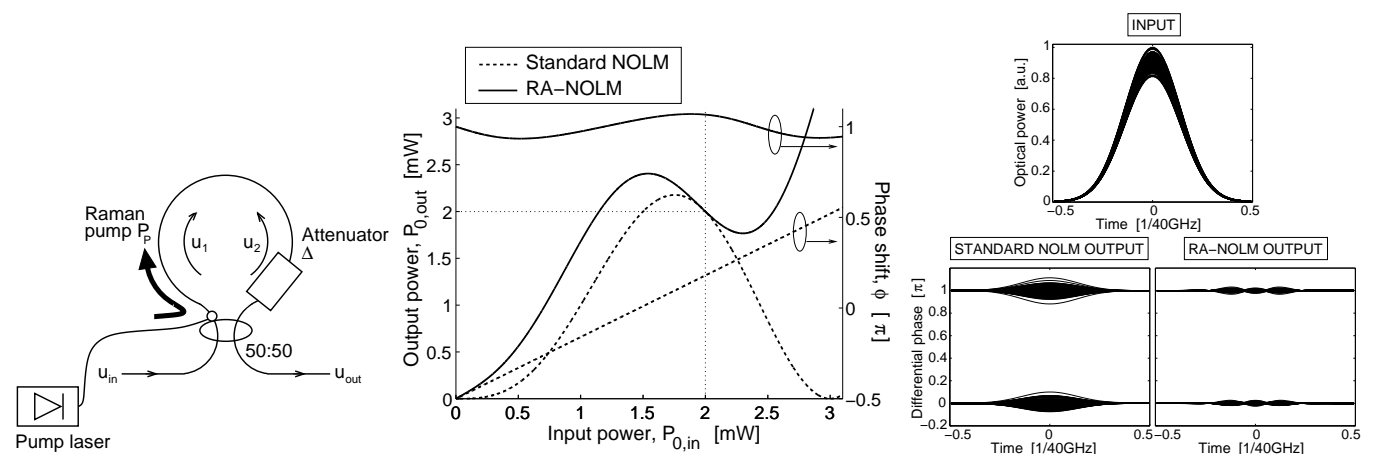

Fig. 16. Left, scheme of the RA-NOLM. Center, CW power and phase characteristics of the standard NOLM and the RA-NOLM. Right, optical DPSK signal eye-diagram at the NOLM input and differential-phase eye-diagrams at the output of the standard NOLM and the RA-NOLM.

The proposed design of RA-NOLM is depicted in Fig. 16 (left). It includes a 50:50 coupler, a loop of fiber with distributed Raman amplification, and an attenuator asymmetrically placed in the loop close to the loop coupler. The Raman pump is coupled into one end of the loop fiber immediately following the loop coupler. Here and hereafter, indexes 1 and 2 refer to the respective clockwise- and counterclockwise-propagating signals. The clockwisepropagating signal is pumped codirectionally such that the gain decreases along the fiber and attenuated by the attenuator after passing the fiber. The counterclockwise-propagating signal is attenuated before passing the fiber and pumped counterdirectionally with increasing gain along the fiber. The different gains "seen" while propagating through the fiber in conjunction with the discrimination provided by the attenuator make the two signals acquire different SPM-induced phase shifts. The inteference of the signals at the coupler yield the nonlinear power transfer function and phase characteristic, $t(P) \equiv\left|u_{\text {out }} / u_{\text {in }}\right|^{2}$ and $\phi(P) \equiv \arg \left(u_{\text {out }} / u_{\text {in }}\right),[84]$

$$
\begin{gathered}
t(P)=\Delta \exp \left(c_{1}+c_{2}\right)\left\{\sinh ^{2}\left[\left(c_{1}-c_{2}\right) / 2\right]+\sin ^{2}\left[\sigma\left(L_{\mathrm{eff}, 1}-\Delta L_{\mathrm{eff}, 2}\right) P / 4\right]\right\} \\
\phi(P)=\sigma\left(L_{\mathrm{eff}, 1}+\Delta L_{\mathrm{eff}, 2}\right) P / 4 \\
+\arctan \left\{\operatorname{coth}\left[\left(c_{1}-c_{2}\right) / 2\right] \tan \left[\sigma\left(L_{\mathrm{eff}, 1}-\Delta L_{\mathrm{eff}, 2}\right) P / 4\right]\right\}
\end{gathered}
$$

Here, $\sigma, \Delta$ and $L$ denote the same quantities as in (1), $c_{1,2}=\int_{0}^{L} \mathrm{~d} z g_{1,2}(z)$, 
$L_{\text {eff }, 1}=\int_{0}^{L} \mathrm{~d} z \exp \left(2 \int_{0}^{z} \mathrm{~d} z^{\prime} g_{1}\left(z^{\prime}\right)\right), L_{\text {eff }, 2}=\int_{0}^{L} \mathrm{~d} z \exp \left(2 \int_{z}^{L} \mathrm{~d} z^{\prime} g_{2}\left(z^{\prime}\right)\right), g_{1}(z)=$ $-\gamma+g_{0} \exp \left(-2 \gamma_{\mathrm{P}} z\right), g_{2}(z)=-\gamma+g_{0} \exp \left(-2 \gamma_{\mathrm{P}}(z-L)\right), \gamma=0.05 \ln (10) \alpha$ and $\gamma_{\mathrm{P}}=0.05 \ln (10) \alpha_{\mathrm{P}}$ are the fiber loss coefficients at the respective signal and pump wavelengths, $g_{0}=g_{\mathrm{R}} P_{\mathrm{P}} /\left(2 A_{\text {eff }}\right), g_{\mathrm{R}}$ is the Raman gain coefficient at the signal wavelength, $A_{\text {eff }}$ is the fiber effective area, and $P_{\mathrm{P}}$ is the injected pump power. The combined action of differential Raman amplification and attenuation-imbalancing within the loop is responsible for an oscillatory behaviour of the induced phase shift $\phi$ around a mean value, whose variation with the input power decreases with decrease of $\Delta$, for fixed loop length and fiber parameters. Using the first equation in (6), the requirements of NOLM operation such that the peak power $P_{0 \text {,in }}$ of the input pulses is in the region past the first peak of the power characteristic and the output pulse peak power equalizes the input one yield $\sigma\left(L_{\mathrm{eff}, 1}-\Delta L_{\mathrm{eff}, 2}\right) P_{0, \text { in }} / 2=(1+\epsilon) \pi, 0<\epsilon<1$, and $\Delta \exp \left(c_{1}+c_{2}\right)\left\{\sinh ^{2}\left[\left(c_{1}-c_{2}\right) / 2\right]+\cos ^{2}(\epsilon \pi / 2)\right\}=1$. The solution of these equations for given $P_{0 \text {,in }}$ and $\epsilon$, and fixed fiber parameters and Raman pump, provides the values of the loop length $L$ and loss coefficient $\Delta$ that minimize the variation of the induced phase shift with the input power. Note that further reduction of the phase-shift variation could be achieved by incorporation of a postamplifier into the NOLM setup. However, this would make the NOLM configuration more complex while yielding negligible performance improvement. Exemplary CW power and phase-characteristic curves according to (6) and are plotted in Fig. 16 (center). The CW power and phase characteristics of an equivalent conventional NOLM (see (1)) are also plotted for comparison. Note that the reduction of the phase-shift variation with the input power in the RA-NOLM compared with the conventional NOLM is achieved at the expenses of a weaker discrimination of the low-power radiation against the higher-power signals. Indeed, it can be easily shown by use of the demanded conditions on the NOLM operation that both types of NOLM attenuate the low input powers $P$ satisfying $P / P_{0, \text { in }}<2 \arcsin [\cos (\epsilon \pi / 2)] /[(1+\epsilon) \pi]$ for fixed $P_{0 \text {,in }}$ and $\epsilon$. However, while the conventional NOLM approximately cubes the low powers, the strength of intensity filtering of the RA-NOLM is determined by the interplay between a linear term and a cubic term with $P$ in the power mapping equation (see (6)). In our calculations, the Raman gain of the loop fiber is varied by varying the loop length $L$ to achieve the desired power and phase responses. Equivalently, one could vary the Raman pump power $P_{\mathrm{P}}$ at a fixed loop length. A rather fine tuning of the Raman gain is required for the operational conditions on the power to be met, whereas a variation in the Raman gain does not change the phase response significantly. Indeed, a deviation of the loop length or pump power from its nominal value will only slightly affect the phase response in that it will cause a slight decrease (increase) of the amplitude of oscillations of the phase shift $\phi$ around its mean value. On the other hand, as it can be seen from Eq. (6), the period of oscillations of both power and phase responses will shorten (lengthen). As a consequence of the variation in the period of the power response, the operational point on 
the power characteristic will shift with respect to the first peak. Moreover, postamplification (postattenuation) of the NOLM will be required for inputoutput power equalization. Note, however, that the sensitivity of the NOLM power response to the effective length of the NOLM loop is a common feature to any NOLM setup.

An illustrative example of the NOLM action on DPSK signals is given in Fig. 16 (right), which shows the optical eye-diagram of a 40-Gb/s RZ-DPSK signal with a random (Gaussian-distributed) jitter of the peak power at the NOLM input, and the eye-diagrams of the signal differential phase at the output of the standard NOLM and the RA-NOLM. It is seen that the conventional NOLM converts the input power fluctuation into proportional fluctuations of the differential phase on the zero and $\pi$ rails. This is due to the linear variation of the phase shift $\phi$ induced by the conventional NOLM with the input power. On the other hand, amplitude jitter causes only low excess phase jitter in the RA-NOLM. Note that an exisiting phase jitter at the NOLM input is not reduced by the proposed NOLM setup.

\subsection{0-Gb/s RZ-DPSK transmission}

Simultaneous amplitude regeneration and suppression of the phase noise growth is highly desirable for in-line DPSK signal processing. Combining the RANOLM with a narrow OBPF into an in-line limiter, it is possible to achieve $2 \mathrm{R}$ regeneration and substantial reduction of the accumulated phase noise simultaneously [84]. Indeed, the RA-NOLM reshapes and stabilizes the signal envelopes while inducing only a minor excess phase fluctuation. The stabilization of amplitude fluctuations leads to reduction of the nonlinear accumulation of the phase noise caused by the amplitude-to-phase noise translation. The narrow filter provides a phase-limiting function because it discriminates the ASE noise generated by in-line amplifiers from the signal and, thus, suppresses the ASE-induced phase noise (linear and nonlinear), which is proportional to the ASE noise power.

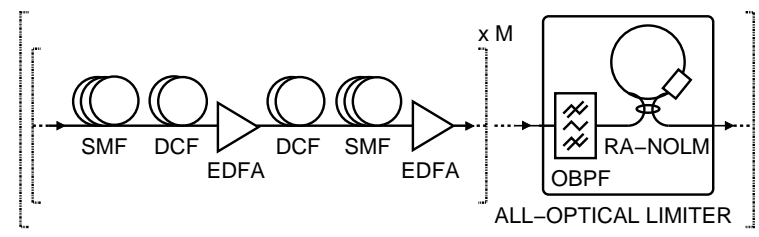

Fig. 17. Unit cell of a periodic transmission line with NOLM-based limiters.

Here, for the purposes of illustration, simulation of a single-channel $40-\mathrm{Gb} / \mathrm{s}$ RZ-DPSK transmission is performed. We model a DM SMF transmission link with an all-optical limiter inserted every $M$ dispersion map periods, as depicted in Fig. 17. The limiter consists of a Gaussian OBPF followed by an 


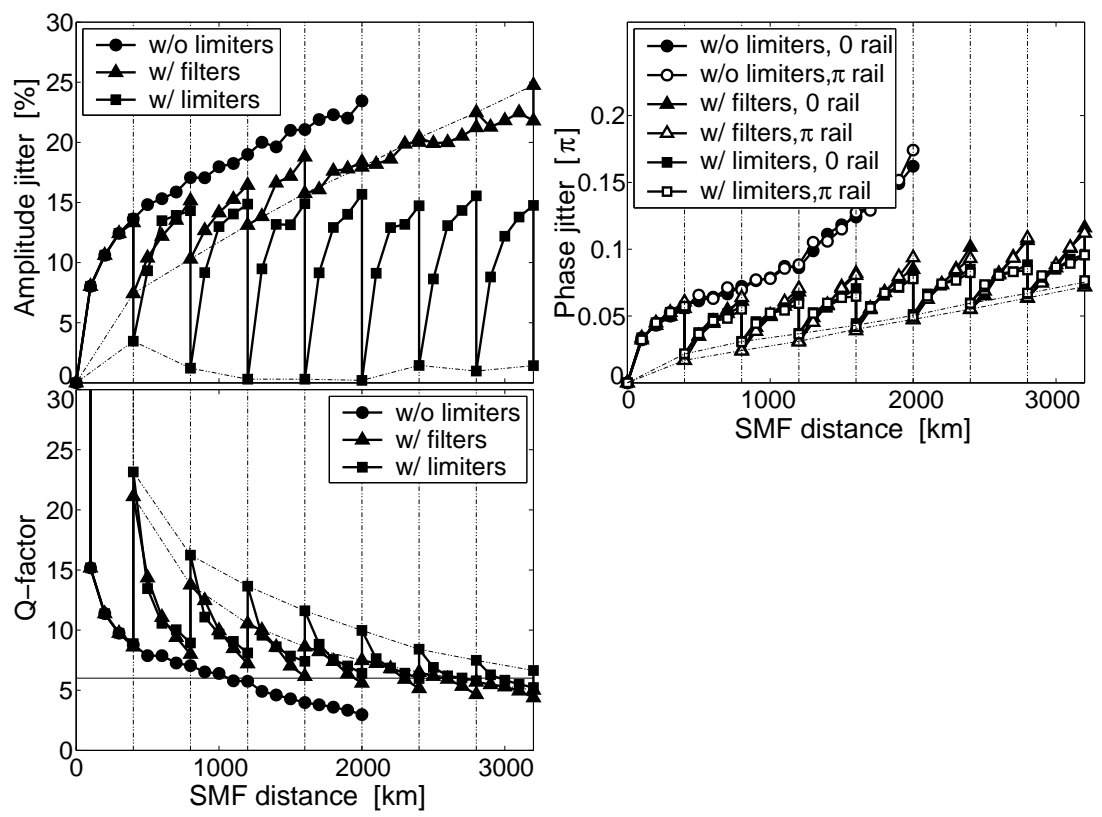

Fig. 18. Amplitude and phase jitters and $Q$-factor versus distance for $40-\mathrm{Gb} / \mathrm{s}$ RZ-DPSK transmission.

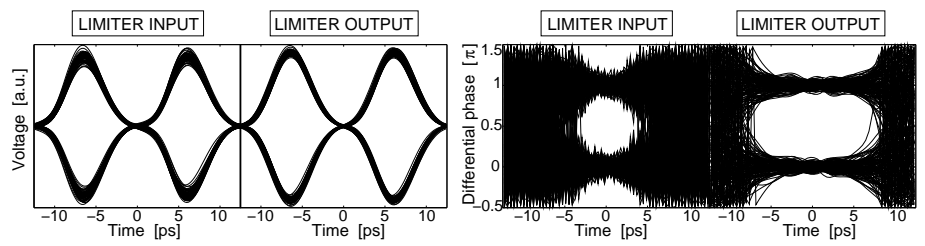

Fig. 19. Electrical signal eye-diagrams and differential phase eye-diagrams before and after the limiter at $1200 \mathrm{~km}$ of SMF.

RA-NOLM. We refer to [84] for details of the system and numerical modeling. Figure 18 shows the evolution of the RMS variations of the signal amplitude and phase and the $Q$-factor over the transmission distance [84]. In this example, $M=4$ (limiter insertion period of $400 \mathrm{~km}$ of SMF). The $Q$-factor is calculated as the instantaneous (i.e., at each distance along transmission) minimum between the differential phase $Q$-factor [132], which provides a measure of the phase fluctuation in the signal, and the conventional $Q$-factor of the demodulated OOK signal, which accounts for the contribution to the performance degradation originating from amplitude noise and/or timing jitter. The evolutions of the RMS amplitude and phase variations and the $Q$-factor for RZ-DPSK signals transmitted through the system without limiters and the system with OBPFs only are also plotted in Fig. 18. It is seen that, in the absence of limiters, there is an approximately linear increase in both the amplitude and phase jitters. For the system with OBPFs only, the filter action in discriminating the ASE noise from the signal results into a substantial suppression of the accumulated phase jitter at each filter location. However, although the effective rate of growth of the amplitude noise in the system keeps lower compared to the case without limiters because of some stabiliza- 
tion of the pulse energy provided by the filter control, the system is eventually dominated by amplitude noise. On the other hand, in the presence of limiters (OBPF + RA-NOLM), the accumulated amplitude jitter is almost completely suppressed at each limiter location by the amplitude regeneration action of the NOLM. Significant suppression of the accumulated phase jitter is also observed at each limiter location due to the filter action, although the strength of phase noise reduction is slightly lower compared to the case with filters only. The latter is due to the excess phase fluctuation introduced by the NOLM. Phase noise is the main limiting factor for such a system, but still at $3,200 \mathrm{~km}$ of SMF the accumulation of phase noise is not enough to produce degradation of the $Q$-factor below six. The regenerative capabilities of the limiter can be seen in Fig. 19 [84], which shows the eye-diagrams of the electrical signal and the differential phase eye-diagrams at the input and the output of the limiter located at $1,200 \mathrm{~km}$ of SMF.

\section{Conclusion}

We have reported on some examples of all-optical nonlinear signal processing techniques based on the fiber NOLM for optical communication systems. Firstly, we have revisited the application of in-line NOLMs as a general technique for all-optical passive $2 \mathrm{R}$ regeneration of RZ-OOK signals in both highspeed, ultralong-distance transmission systems and terrestrial photonic networks. Specifically, we have reported a numerical demonstration of the feasibility of single-channel data transmissions over virtually unlimited distances at $40 \mathrm{~Gb} / \mathrm{s}$ in standard fiber systems where the transmission performance is mainly degraded by amplitude noise and waveform distortion. We have described the extension of the regeneration technique to dense WDM transmissions at $40 \mathrm{~Gb} / \mathrm{s}$ per channel over ultralong distances. We have discussed the application of the technique in all-optical WDM networks at 40-Gb/s channel rate.

Secondly, we have presented a theoretical model enabling the description of the ultrashort pulse propagation in fiber transmission systems in the quasilinear regime with periodic in-line deployment of nonlinear optical devices. In the particular application to NOLMs, we have demonstrated that autosoliton formation can be observed in such systems as a result of a balance between the effects of dispersion in the transmission fibers, linear control by optical filters, and nonlinear focusing in the NOLMs. We have applied an approximate variational approach to determine the steady-state pulse characteristics and have shown that the theoretical analysis accurately reproduces the numerical results.

Thirdly, we have described a simple all-optical nonlinear pulse processing tech- 
nique that exploits the Kerr effect in a NDF for improvement of the phase margin of the optical signals and the intensity filtering action of a NOLM for achieving signal reamplification and cleaning up. We have demonstrated the use of such a pulse processor as an all-optical decision element at a RZ optical receiver at $40 \mathrm{~Gb} / \mathrm{s}$ to improve the signal quality before detection. We have furthermore shown an in-line employment of the device in the realization of a transmission technique of all-optical periodic format conversion between RZ and NRZ-like at $40 \mathrm{~Gb} / \mathrm{s}$.

Finally, we have presented a concept for phase-preserving all-optical regeneration of signals using phase-encoded modulation formats, which exploits a novel design of NOLM based on distributed Raman amplification in the loop fiber. The combined use of the RA-NOLM and optical filtering as an in-line limiter into a 40-Gb/s RZ-DPSK system has been demonstrated to provide simultaneous $2 \mathrm{R}$ regeneration and phase noise suppression.

\section{Acknowledgements}

We would like to acknowledge and to thank our co-authors of the publications that form a basis for this review paper, viz. Dr. Stanislav A. Derevyanko, Prof. Alexander S. Kovalev, Prof. Mikhail M. Bogdan, and Dr. Ranjeet Bhamber.

S. Boscolo gratefully acknowledges the Leverhulme Trust for supporting her work through an Early Career Fellowship (Ref. No. ECF/2005/0400).

\section{References}

[1] O. Leclerc, B. Lavigne, D. Chiaroni, and E. Desurvire, All-optical regeneration: Principles and WDM implementation, in: Optical Fiber Telecommunications IVA: Components, I. Kaminov, T. Li, eds (Academic, 2002), pp. 732-783.

[2] O. Leclerc, B. Lavigne, E. Balmefrezol, P. Brindel, L. Pierre, D. Rouvillain, and F. Seguineau, Optical regeneration at $40 \mathrm{~Gb} / \mathrm{s}$ and beyond, J. Lightwave Technol. 21 (2003), 2779-2790.

[3] D. Wolfson, P. B. Hansen, A. Kloch, and K. E. Stubkjaer, All-optical 2R regeneration based on interferometric structure incorporating semiconductor optical amplifiers, Electron. Lett. 35 (1999), 59-60.

[4] K. E. Stubkjaer, Semiconductor optical amplifier-based all-optical gates for high-speed optical processing, IEEE J. Sel. Top. Quantum Electron. 6 (2000), 1428-1435. 
[5] D. Wolfson, A. Kloch, T. Fjelde, C. Janz, B. Dagens, and M. Renaud, 40-Gb/s all-optical wavelength conversion, regeneration, and demultiplexing in an SOAbased all-active Mach-Zehnder interferometer, IEEE Photon. Technol. Lett. 12 (2000), 332-334.

[6] K. Tajima, S. Nakamura, and Y. Ueno, Ultra-fast all-optical signal processing with symmetric Mach-Zehnder type all-optical switches, Opt. Quantum Electron. 33 (2001), 875-897.

[7] Y. Ueno, S. Nakamura, and K. Tajima, Penalty-free error-free all-optical pulse regeneration at $84 \mathrm{~Gb} / \mathrm{s}$ by using a symmetric Mach-Zehnder type semiconductor regenerator, IEEE Photon. Technol. Lett. 13 (2001), 469-471.

[8] N. S. Patel, K. A. Rouschenbach, and K. L. Hall, 40-Gbps demultiplexing using an ultrafast nonlinear interferometer (UNI), IEEE Photon. Technol. Lett. 8 (1996), 1695-1697.

[9] J. Leuthold, B. Mikkelsen, R. E. Behringer, G. Raybon, C. H. Joyner, and P. A. Besse, Novel 3R regenerator based on semiconductor optical amplifier delayedinterference configuration, IEEE Photon. Technol. Lett. 13 (2001), 860-862.

[10] C. Bornholdt, J. Slovak, and B. Sartorius, Semiconductor-based all-optical 3R regenerator demonstrated at $40 \mathrm{Gbit} / \mathrm{s}$, Electron. Lett. 40 (2004), 192-194.

[11] O. Audoin, E. Pallise, and E. Desurvire, Signal stability in periodically amplified fiber transmission systems using multiple quantum well saturbale absorbers for regeneration, IEEE Phton. Technol. Lett. 10 (1998), 828-830.

[12] D. Rouvillain, P. Brindel, E. Seguineau, L. Pierre, O. Leclerc, H. Choumane, G. Aubin, and J.-L. Oudar, Optical 2R regenerator based on passive saturable absorber for $40 \mathrm{Gbit} / \mathrm{s}$ WDM long-haul transmissions, Electron. Lett. 38 (2002), $1113-1114$.

[13] T. Miyazaki, T. Otani, N. Edagawa, M. Suzuki, and S. Yamamoto, Novel optical regenerator using electroabsorption modulator, IEICE Tans. Electron. E82-C (1999), 1414-1419.

[14] E. Ciaramella, F. Curti, and S. Trillo, All-optical signal reshaping by means of four-wave mixing in optical fibers, IEEE Photon. Technol. Lett. 13 (2001), $142-144$.

[15] Y. Su, L. Wang, A. Agrawal, and P. Kumar, All-optical limiter using gain flattened fiber parametric amplifier, Electron. Lett. 36 (2000), 1103-1105.

[16] , S. Radic, C. J. McKinstrie, R. M. Jopson, J. C. Centanni, and A. R. Charplyvy, All-optical regeneration in one- and two-pump parametric amplifiers using highly nonlinear optical fiber, IEEE Photon. Technol. Lett. 15 (2003), 957-959.

[17] A. Bogris and D. Syvridis, Regenerative properties of a pump-modulated fourwave mixing scheme in dispersion-shifted fibers, J. Lightwave Technol. 21 (2003), 1892-1902. 
[18] S. Yamashita and M. Shahed, Optical 2R regeneration using cascaded fiber fourwave mixing with suppressed spectral spread, IEEE Photon. Technol. Lett. 18 (2006), 1064-1066.

[19] P. V. Mamyshev, All-optical data regeneration based on self-phase modulation effect, in: Proc. European Conference on Optical Communications (ECOC'98), Madrid, Spain, 1998, pp. 475-476.

[20] G. Raybon, Y. Su, J. Leuthold, R. Essiambre, T. Her, P. Steinvurzel, K. Dreyer, K. Feder, and C. Joergensen, $40 \mathrm{Gbit} / \mathrm{s}$ pseudo-linear transmission over one million kilometers, in: Proc. Optical Fiber Communication Conference (OFC'02), Anaheim, California, 2002, PD-paper FD10.

[21] N. Yoshikane, I. Morita, T. Tsuritani, A. Agata, N. Edagawa, and S. Akiba, Benefit of SPM-based all-optical reshaper in receiver for long-haul DWDM transmission systems, IEEE J. Sel. Top. Quantum Electron. 10 (2004), 412420 .

[22] A. G. Striegler and B. Schmauss, Analysis and optimization of SPM-based 2R signal regeneration at $40 \mathrm{~Gb} / \mathrm{s}$, J. Lightwave Technol. 24 (2006), 2835-2843.

[23] M. Rochette, J. L. Blows, and B. J. Eggleton, 3R optical regeneration: an alloptical solution with BER improvement, Opt. Express 14 (2006), 6414-6427.

[24] L. Provost, C. Finot, P. Petropoulos, K. Mukasa, and D. J. Richardson, Design scaling rules for 2R-optical self-phase modulation-based regenerators, Opt. Express 15 (2007), 5100-5113.

[25] T. I. Lakoba and M. Vasilyev, A new robust regime for a dispersion-managed multichannel 2R regenerator, Opt. Express 15 (2007), 10061-10074.

[26] A. Sahara, T. Inui, T. Komukai, H. Kubota, and M. Nakazawa, 40-Gb/s RZ transmission over transoceanic distance in a dispersion managed standard fiber using a new inline synchronous modulation method, IEEE Photon. Technol. Lett. 13 (2001), 1248-1250.

[27] M. Matsumoto and O. Leclerc, Analysis of 2R optical regenerator utilizing selfphase modulation in highly nonlinear fiber, Electron. Lett. 38 (2002), 576-577.

[28] M. Matsumoto, Performance analysis and comparison of optical 3R regenerators utilizing self-phase modulation in fibers, J. Lightwave Technol. 22 (2004), 14721482 .

[29] J. Yu and P. Jeppesen, Simultaneous all-optical demultiplexing and regeneration based on self-phase and cross-phase modulation in a dispersion shifted fiber, J. Lightwave Technol. 19 (2001), 941-949.

[30] S. Boscolo, J. H. B. Nijhof, and S. K. Turitsyn, Autosoliton transmission in dispersion-managed systems guided by in-line nonlinear optical loop mirrors, Opt. Lett. 25 (2000), 1240-1242.

[31] S. Boscolo, S. K. Turitsyn, and K. J. Blow, All-optical passive regeneration of $40 \mathrm{Gbit} / \mathrm{s}$ soliton data stream using dispersion management and in-line nonlinear optical loop mirrors, Electron. Lett. 37 (2001), 112-113. 
[32] K. J. Blow, S. Boscolo, and S. K. Turitsyn, Optical pulse regeneration using dispersion managed optical fibre and an unbalanced interferometer, Patent GB2371160, 17 Jul. 2002. Optical pulse regenerating transmission lines, Patent WO02056506, 18 Jul. 2002; Patent EP1356612, 29 Oct. 2003; Patent US2004076373, 22 Apr. 2004.

[33] S. Boscolo, S. K. Turitsyn, and K. J. Blow, Study of the operating regime for all-optical $2 \mathrm{R}$ regeneration of dispersion-managed $\mathrm{RZ}$ data at $40 \mathrm{~Gb} / \mathrm{s}$ using in-line NOLMs, IEEE Photon. Technol. Lett. 14 (2002), 30-32.

[34] S. Boscolo, S. K. Turitsyn, and K. J. Blow, All-optical passive quasi-regeneration in transoceanic $40 \mathrm{Gbit} / \mathrm{s}$ return-to-zero transmission systems with strong dispersion management, Opt. Commun. 205 (2002), 277-280.

[35] S. Boscolo, S. K. Turitsyn, K. J. Blow, and J. H. B. Nijhof, Passive regeneration in $40 \mathrm{Gbit} / \mathrm{s}-$ based WDM dispersion-managed RZ transmission systems by inline NOLMs, Opt. Fiber Technol. 8 (2002), 313-318.

[36] S. Boscolo, S. K. Turitsyn, and K. J. Blow, All-optical passive 2R regeneration for $N \times 40 \mathrm{Gbit} / \mathrm{s}$ WDM transmission using NOLM and novel filtering technique, Opt. Commun. 217 (2003), 227-232.

[37] S. Boscolo, K. J. Blow, and S. K. Turitsyn, All-optical 2R regeneration in scalable 40-Gbit/s wavelength-division multiplexed networks, J. Opt. Networking (2003), 10-16.

[38] S. Boscolo, S. K. Turitsyn, and V. K. Mezentsev, Performance comparison of $2 \mathrm{R}$ and $3 \mathrm{R}$ optical regeneration schemes at $40 \mathrm{~Gb} / \mathrm{s}$ for application to all-optical networks, IEEE J. Lightwave Technol. 23 (2005), 304-309.

[39] F. Seguineau, B. Lavigne, D. Rouvillain, L. Pierre, P. Brindel, and O. Leclerc, Experimental demonstration of simple NOLM-based 2R regenerator for $42.66 \mathrm{Gbit} / \mathrm{s}$ WDM long-haul transmissions, in: Proc. Optical Fiber Communication Conference (OFC'04), Anaheim, California, 2004, paper WN4.

[40] M. Meissner, M. Rösch, B. Schmauss, and G. Leuchs, Optimum splitting ratio for amplifier noise reduction by an asymmetric nonlinear optical loop mirror, Appl. Phys. B 80 (2005), 489-495.

[41] M. Meissner, M. Rösch, B. Schmauss, and G. Leuchs, $12 \mathrm{~dB}$ of noise reduction by a NOLM-based 2-R regenerator, IEEE Photon. Technol. Lett. 15 (2003), $1297-1299$.

[42] M. Meissner, K. Sponsel, K. Cvecek, A. Benz, S. Weissner, B. Schmauss, and G. Leuchs, $3.9 \mathrm{~dB}$ OSNR gain by an NOLM-based 2-R regenerator, IEEE Photon. Technol. Lett. 16 (2004), 2105-2107.

[43] A. G. Striegler and B. Schmauss, Extinction ratio improvement by an advanced NOLM setup, IEEE Photon. Technol. Lett. 18 (2006), 1058-1060.

[44] N. Chi, L. Xu, L. Oxenløwe, T. Tokle, and P. Jeppesen, 2R regenerator based on high non-linear dispersion imbalanced loop mirror, Opt. Commun. 206 (2002), 295-300. 
[45] N. Chi, B. Carlsson, and P. Jeppesen, 2R regeneration based on dispersionimbalanced loop mirror and its applications in WDM systems, J. Lightwave Technol. 20 (2002), 1809-1817.

[46] N. Chi, L. Xu, K. S. Berg, T. Tokle, and P. Jeppesen, All-optical wavelength conversion and multichannel $2 \mathrm{R}$ regeneration based on highly nonlinear dispersion-imbalanced loop mirror, IEEE Photon. Technol. Lett. 14 (2002), $1581-1583$.

[47] A. Bogoni, P. Ghelfi, M. Scaffardi, and L. Potì, All-optical regeneration and demultiplexing for $160-\mathrm{Gb} / \mathrm{s}$ transmission systems using NOLM-based three stage scheme, IEEE J. Sel. Top. Quantum Electron. 10 92004), 192-196.

[48] S. Watanable and F. Futami, All-optical signal processing using highlynonlinear optical fibers, IEICE Trans. Commun. E84-B (2001), 1179-1189.

[49] N. J. Doran and D. Wood, Nonlinear-optical loop mirror, Opt. Lett. 13 (1988), $56-58$.

[50] K. J. Blow, N. J. Doran, and B. K. Nayar, Experimental demonstration of optical soliton switching in an all-fiber nonlinear Sagnac interferometer, Opt. Lett. 14 (1989), 754-756.

[51] M. N. Islam, E. R. Sunderman, R. H. Stolen, W. Pleibel, and J. R. Simpson, Soliton switching in a fiber nonlinear loop mirror, Opt. Lett. 14 (1989), 811-813.

[52] D. A. Pattison, W. Forysiak, P. N. Kean, I. Bennion, and N. J. Doran, Soliton switching using cascaded nonlinear-optical loop mirrors, Opt. Lett. 20 (1995), $19-21$.

[53] W. S. Wong, S. Namiki, M. Margalit, H. A. Haus, and E. P. Ippen, Self-switching of optical pulses in dispersion-imbalanced nonlinear loop mirrors, Opt. Lett. 22 (1997), 1150-1152.

[54] O. Pottiez, E. A. Kuzin, B. Ibarra-Escamilla, J. L. Camas-Anzueto, and F. Gutierrez-Zainos, Experimental demonstration of NOLM switching based on nonlinear polarization rotation, Electron. Lett. 40 (2004), 892-894.

[55] W. Cao and P. K. A. Wai, Comparison of fiber-based Sagnac interferometers for self-switching of optical pulses, Opt. Commun. 245 (2005), 177-186.

[56] H. C. Lim, F. Futami, and K. Kikuchi, Polarisation independent wavelengthshift-free optical phase conjugator using a nonlinear fiber Sagnac interferometer, IEEE Photon. Technol. Lett. 11 (1999), 578-580.

[57] D. M. Patrick, A. D. Ellis, and D. M. Spirit, Bit-rate flexible all-optical demultiplexing using a nonlinear optical loop mirror, Electron. Lett. 29 (1993), 702-703.

[58] Y. Liang, J. W. Lou, J. C. Stocker, O. Boyraz, J. K. Andersen, M. N. Islam, and D. A. Nolam, Polarization-insensitive nonlinear optical loop mirror demultiplexer with twisted fiber, Opt. Lett. 24 (1999), 726-728 
[59] H. Sotobayashi, C. Sawaguchi, Y. Koyamada, and W. Chujo, Ultrafast walk-offfree nonlinear optical loop mirror by a simplified configuration for 320-Gbit/s time-division multiplexing signal demultiplexing, Opt. Lett. 27 (2002), 15551557.

[60] I. N. Duling III and M. L. Dennis, Compact sources of ultrashort pulses (Cambridge University Press, Cambridge, 1995).

[61] E. A. Kuzin, B. Ibarra-Escamilla, D. E. Garcia-Gomez, and J. W. Haus, Fiber laser modelocked by the nonlinear polarization rotation Sagnac interferometer, Opt. Lett. 26 (2001), 1559-1561.

[62] B. Ibarra-Escamilla, E. A. Kuzin, D. E. Gomez-Garcia, F. Gutierrez-Zainos, S. Mendoza-Vasquez, and J. W. Haus, A modelocked fiber laser using a Sagnac interferometer and nonlinear polarization rotation, J. Opt. A: Pure Appl. Opt. 5 (2003), S225-S230.

[63] I. Golub and E. Simova, Ring resonator in a Sagnac interferometer as a birifringence magnifier, Opt. Lett. 30 (2005), 87-89.

[64] E. Simova, I. Golub, and M. J. Picard, Ring resonator in a Sagnac loop, J. Opt. Soc. Am. B 22 (2005), 1723-1730.

[65] K. Smith, N. J. Doran, and P. G. J. Wigley, Pulse shaping, compression, and pedestal suppression employing a nonlinear-optical loop mirror, Opt. Lett. 15 (1990), 1294-1296.

[66] A. L. Steele, Pulse compression by an optical-fiber loop mirror constructed from 2 different fibers, Electron. Lett. 29 (1993), 1971-1972.

[67] M. D. Pelusi, Y. Matsui, and A. Suzuki, Pedestal suppression from compressed femtosecond pulses using a nonlinear fiber loop mirror, IEEE J. Quantum Electron. 35 (1999), 867-874.

[68] K. R. Tamura and M. Nakazawa, A polarization-maintaining pedestal-free femtosecond pulse compressor incorporating an ultrafast dispersion-imbalanced nonlinear optical loop mirror, IEEE Photon. Technol. Lett. 13 (2001), 526-528.

[69] J. H. Lee, T. Kogure, and D. J. Richardson, Wavelength tunable 10-GHz 3-ps pulse source using a dispersion decreasing fiber-based nonlinear optical loop mirror, IEEE J. Quantum Electron. 10 (2004), 181-185.

[70] N. J. Smith and N. J. Doran, Picosecond soliton transmission using concatenated nonlinear optical loop-mirror intensity filters, J. Opt. Soc. Am. B 12 (1995), 1117-1125.

[71] I. Gabitov, D. D. Holm, and B. P. Luce, Low-noise picosecond soliton transmission by use of concatenated nonlinear amplifying loop mirrors, J. Opt. Soc. Am. B 14 (1997), 1850-1855.

[72] I. Gabitov, D. D. Holm, B. P. Luce, and A. Mattheus, Recovery of solitons with nonlinear amplifying loop mirrors, Opt. Lett. 20 (1995), 2490-2492. 
[73] M. Matsumoto, H. Ikeda, and A. Hasegawa, Suppression of noise accumulation in bandwidth-limited soliton transmission by means of nonlinear loop mirrors, Opt. Lett. 19 (1994), 183-185.

[74] M. Matsumoto and A. Hasegawa, Adiabatic amplification of solitons by menas of nonlinear amplifying loop mirrors, Opt. Lett. 19 (1994), 1019-1021.

[75] O. Pottiez, E. A. Kuzin, B. Ibarra-Escamilla, F. Gutiérrez-Zainos, U. RuizCorona, and J. T. Camas-Anzueto, High-order amplitude regularization of an optical pulse train using a power-symmetric NOLM with adjustable contrast, IEEE Photon. Technol. Lett. 17 (2005), 154-156.

[76] O. Pottiez, B. Ibarra-Escamilla, and E. A. Kuzin, High-quality amplitude jitter reduction and extinction enhancement using a power-symmetric NOLM and a polarizer, Opt. Express 15 (2007), 2564-2572.

[77] M. E. Fermann, F. Haberl, M. Hofer, and H. Hochreiter, Nonlinear amplifying loop mirror, Opt. Lett. 15 (1990), 752-754.

[78] S. Boscolo, S. A. Derevyanko, S. K. Turitsyn, A. S. Kovalev, and M. M. Bogdan, Autosoliton propagation and mapping problem in optical fiber lines with lumped nonlinear devices, Phys. Rev. E 72 (2005), 016601(4).

[79] S. Boscolo, S. A. Derevyanko, S. K. Turitsyn, A. S. Kovalev, and M. M. Bogdan, Evolution of optical pulses in fiber lines with lumped nonlinear devices as a mapping problem, Theor. and Math. Phys. 144 (2005), 1117-1127 [Teor. i Mat. Fiz. 144 (2005), 277-289].

[80] S. Boscolo and S. K. Turitsyn, All-optical nonlinear pulse processing based on normal dispersion fiber-enhanced nonlinear optical loop mirror, IEEE Photon. Technol. Lett. 16 (2004), 1912-1914.

[81] S. Boscolo and S. K. Turitsyn, Optical pulse regenerator, Patent WO2005076502, 18 Aug. 2005.

[82] S. Boscolo, S. K. Turitsyn, and K. J. Blow, All-optical nonlinear signal processing at a RZ receiver, in: Optical Networks and Technologies, K.-I. Kitayama, F. Masetti-Placci, G. Prati eds. (Springer, 2004), pp. 416-421.

[83] S. Boscolo and S. K. Turitsyn, Optical data transmission using periodic in-line all-optical format conversion, Opt. Express 12 (2004), 4875-4880.

[84] S. Boscolo, R. Bhamber, and S. K. Turitsyn, Design of Raman-based nonlinear loop mirror for all-optical $2 \mathrm{R}$ regeneration of differential phase-shift keying transmission, IEEE J. Quantum Electron. 42 (2006), 619-624.

[85] I. Shake, H. Takara, K. Uchiyama, S. Kawanishi, and Y. Yamabayashi, Vibration-insensitive nonlinear optical loop mirror utilizing reflecting scheme, IEEE Photon. Technol. Lett. 12 (2000), 555-557.

[86] R.-J. Essiambre, B. Mikkelsen, and G. Raybon, Intra-channel cross-phase modulation and four-wave mixing in high-speed TDM systems, Electron. Lett. 35 (1999), 1576-1578. 
[87] R.-J. Essiambre, G. Raybon, and B. Mikkelsen, Pseudo-linear transmission of high-speed signals: 40 and $160 \mathrm{Gbit} / \mathrm{s}$, in: Optical Fiber Telecommunications IVB, I. P. Kaminow, T. Li eds. (Academic, New Jersey, 2002), pp. 232-304.

[88] P. V. Mamyshev and N. A. Mamysheva, Pulse-overlapped dispersion-managed data transmission and intrachannel four-wave mixing, Opt. Lett. 24 (1999), $1456-1458$.

[89] A. Mecozzi, C. B. Clausen, and M. Shtaif, Analysis of intrachannel nonlinear effects in highly dispersed optical pulse transmission, IEEE Photon. Technol. Lett. 12 (2000), 392-394.

[90] G. P. Agrawal, Fiber-Optic Communication Systems (3rd ed., Wiley, New York, 2002).

[91] S. Bigo, Improving spectral efficiency by ultra-narrow optical filtering to achieve multiterabit/s capacities, In: Proc. Optical Fiber Communication Conference (OFC'02), Anaheim, California, 2002, paper WX3, pp. 362-364.

[92] B. S. Kerner and V. V. Osipov, Autosolitons: A New Approach to Problems of Self-Organization and Turbolence (Kluwer, Dordrecht, 1994).

[93] Dissipative Solitons, Series: Lecture Notes in Physics 661, N. Akhmediev, A. Ankiewicz eds. (Springer, 2005).

[94] V. S. Grigoryan, Autosolitons in inverted media, Phys. Rev. A 149 (1990), 371-380.

[95] A. G. Vladimirov, G. V. Khodova, and N. N. Rosanov, Stable bound states of one-dimensional autosolitons in a bistable laser, Phys. Rev. E 63 (2001), 056607(6).

[96] A. Hasegawa and Y. Kodama, Solitons in Optical Communications (Oxford University Press, Oxford, 1995).

[97] I. Gabitov and S. K. Turitsyn, Averaged pulse dynamics in cascaded transmission system with passive dispersion compensation, Opt. Lett. 21 (1996), $327-329$.

[98] M. Suzuki, H. Toda, A. H. Liang, and A. Hasegawa, Improvement of amplitude and phase margins in an RZ optical receiver using Kerr nonlinearity in normal dispersion fiber, IEEE Photon. Technol. Lett. 13 (2001), 1248-1250.

[99] P. Ghelfi, M. Secondini, M. Scaffardi, F. Fresi, A. Bogoni, and L. Potì, Impact of an additional all-optical decision element in band-limited receivers for RZ systems, J. Lightwave Technol. 25 (2007), 1728-1734.

[100] P. Ghelfi, M. Scaffardi, A. Bogoni, and L. Potì, Optical receiver scheme with all-optical decision element, Patent WO/2007/039225, 12 Apr. 2007.

[101] S. Boscolo, S. K. Turitsyn, and K. J. Blow, Time domain all-optical signal processing at a RZ optical receiver, Opt. Express 13 (2005), 6217-6227. 
[102] I. O. Nasieva, S. Boscolo, and S. K. Turitsyn, Bit error rate improvement by nonlinear optical decision element, Opt. Lett. 31 (2006), 1205-1207.

[103] M. Matsuura, N. Kishi, and T. Miki, Performance improvement of optical RZreceiver by utilizing an all-optical waveform converter, Opt. Express 13 (2005), 5074-5079.

[104] H. Nakatsuka, D. Grischkowsky, and A. C. Balant, Nonlinear picosecond-pulse propagating through optical fibers with positive group velocity dispersion, Phys. Rev. Lett. 47 (1981), 910-913.

[105] B. Bakhshi, P. A. Andrekson, M. Karlsson, and K. Bertilsson, Soliton interaction penalty reduction by receiver filtering, IEEE Photon. Technol. Lett. 10 (1998), 1042-1044.

[106] F. Forghieri, R. W. Tkach, and A. R. Chraplyvy, Fiber nonlinearities and their impact on transmission systems, in: Optical Fiber Telecommunications IIIA, I. P. Kaminow, T. L. Koch eds. (Academic, San Diego, 1997), pp. 196-264.

[107] L. F. Mollenauer, J. P. Gordon, and P. V. Mamyshev, Solitons in high bitrate, long-distance transmission, in: Optical Fiber Telecommunications IIIA, I. P. Kaminow, T. L. Koch eds. (Academic, San Diego, 1997), pp. 373-460.

[108] E. Iannone, F. Matera, A. Mecozzi, and M. Settembre, Nonlinear Optical Communication Networks (John Wiley \& Sons, 1998).

[109] D. Breuer and K. Petermann, Comparison of NRZ- and RZ- modulation format for $40 \mathrm{Gbit} / \mathrm{s}$ TDM standard-fiber systems, IEEE Photon. Technol. Lett. 9 (1997), 398-400.

[110] K. S. Cheng and J. Conradi, Reduction of pulse-to-pulse interaction using alternative RZ formats in 40-Gb/s system, IEEE Photon. Technol. Lett. 14 (2002), 98-100.

[111] X. Liu, X. Wei, A. H. Gnauck, C. Xu, and I. K. Wickham, Suppression of intrachannel four-wave-mixing-induced ghost pulses in high-speed transmission by phase inversion between adjacent marker blocks, Opt. Lett. 27 (2002), 11771179 .

[112] A. V. Kanaev, G. G. Luther, V. Kovanis, S. R. Bickham, and J. Conradi, Ghost-pulse generation suppression in phase-modulated 40-Gb/s RZ transmission, J. Lightwave Technol. 21 (2003), 1486-1489.

[113] S. Bigo, O. Leclerc, and E. Desurvire, All-optical fiber signal processing and regeneration for soliton communications, IEEE J. Sel. Top. Quantum Electron. 3 (1997), 1208-1222.

[114] S. G. Park, L. H. Spiekman, M. Eiselt, and J. M. Wiesenfeld, Chirp consequences of all-optical RZ to NRZ conversion using cross-phase modulation in an active semiconductor photonic integrated circuit, IEEE Photon. Technol. Lett. 12 (2000), 233-235. 
[115] L. Xu, B. C. Wang, V. Baby, I. Glesk, and P. R. Prucnal, All-optical data format conversion between RZ and NRZ based on a Mach-Zehnder interferometric wavelength converter, IEEE Photon. Technol. Lett. 15 (2003), 308-310.

[116] , K. P. Ho, Phase-Modulated Optical Communication Systems (Springer, 2005).

[117] P. J. Winzer and R.-J. Essiambre, Advanced modulation formats for highcapacity optical transport networks, J. Lightwave Technol. 24 (2006), 47114728 .

[118] A. H. Gnauck and P. J. Winzer, Optical phase-shift-keyed transmission, J. Lightwave Technol. 23 (2005), 115-130.

[119] J. P. Gordon and L. F. Mollenauer, Phase noise in photonic communication systems using linear amplifiers, Opt. Lett. 15 (1990), 1351-1353.

[120] H. Kim and A. H. Gnauck, Experimental investigation of the performance limitation of DPSK systems due to nonlinear phase noise, IEEE Photon. Technol. Lett. 15 (2003), 320-322.

[121] K. Croussore, C. Kim, and G. Li, All-optical regeneration of differential phaseshift keying signals based on phase-sensitive amplification, Opt. Lett. 29 (2004), $2357-2359$.

[122] K. Croussore, I. Kim, Y. Han, C. Kim, and G. Li, Demonstration of phaseregeneration of DPSK signals based on phase-sensitive amplification, Opt. Express 13 (2005), 3945-3950.

[123] V. S. Grigoryan, M. Shin, P. Devgan, and P. Kumar, Mechanism of SOA-based regenerative amplification of phase-noise degraded DPSK signals, Electron. Lett. 41 (2005), 1021-1022.

[124] V. S. Grigoryan, M. Shin, P. Devgan, J. Lasri, and P. Kumar, SOA-based regenerative amplification of phase-noise-degraded DPSK signals: dynamic analysis and demonstration, J. Lightwave Technol. 24 (2006), 135-142.

[125] A. G. Striegler and B. Schmauss, All-optical DPSK signal regeneration based on cross-phase modulation, IEEE Photon. Technol. Lett. 16 (2004), 1083-1085.

[126] A. G. Striegler, M. Meissner, K. Cveček, K. Sponsel, G. Leuchs, and B. Schmauss, NOLM-based RZ-DPSK signal regeneration, IEEE Photon. Technol. Lett. 17 (2005), 639-641.

[127] K. Cvecek, G. Onishchukov, K. Sponsel, A. G. Striegler, B. Schmauss, and G. Leuchs, Experimental investigation of modified NOLM for phase-encoded signal regeneration, IEEE Photon. Technol. Lett. 18 (2006), 1801-1803.

[128] K. Cvecek, K. Sponsel, G. Onishchukov, B. Schmauss, and G. Leuchs, 2Rregeneration of a RZ-DPSK signal using a nonlinear amplifying loop mirror, IEEE Photon. Technol. Lett. 19 (2007), 146-148. 
[129] M. Matsumoto, Regeneration of RZ-DPSK signals by fiber-based all-optical regenerators, IEEE Photon. Technol. Lett. 17 (2005), 1055-1057.

[130] M. Matsumoto, Performance improvement of phase-shift-keying signal transmission by means of optical limiters using four-wave mixing in fibers, J. Lightwave Technol. 23 (2005), 2696-2701.

[131] M. Matsumoto and K. Sanuki, Performance improvement of DPSK signal transmission by a phase-preserving amplitude limiter, Opt. Express 15 (2007), 8094-8103.

[132] C. Xu, X. Liu, and X. Wei, Differential phase-shift keying for high spectral efficiency optical transmissions, IEEE J. Sel. Top. Quantum Electron. 10 (2004), $281-293$. 